\title{
Article \\ Potent Antiplasmodial Derivatives of Dextromethorphan Reveal the Ent-Morphinan Pharmacophore of Tazopsine-Type Alkaloids
}

\author{
Antoinette Keita ${ }^{1}\left(\mathbb{D}\right.$, Jean-François Franetich ${ }^{2,+}$, Maëlle Carraz ${ }^{3,+} \mathbb{D}^{\mathbb{D}}$, Loïse Valentin ${ }^{2,4,+} \mathbb{D}$, \\ Mallaury Bordessoules ${ }^{2}$, Ludivine Baron ${ }^{2}$, Pierre Bigeard ${ }^{2}$, Florian Dupuy ${ }^{5,6}$, Valentine Geay ${ }^{7}$, Maurel Tefit ${ }^{2}$, \\ Véronique Sarrasin ${ }^{8}$, Sylvie Michel ${ }^{7}$ D, Catherine Lavazec ${ }^{5,6}$, Sandrine Houzé ${ }^{1,8} \mathbb{D}^{1}$, Dominique Mazier ${ }^{2}$, \\ Valérie Soulard ${ }^{2}$, François-Hugues Porée ${ }^{9, *}$ (D) and Romain Duval ${ }^{1, *}$
}

check for updates

Citation: Keita, A.; Franetich, J.-F.; Carraz, M.; Valentin, L.; Bordessoules, M.; Baron, L.; Bigeard, P.; Dupuy, F.; Geay, V.; Tefit, M.; et al. Potent Antiplasmodial Derivatives of Dextromethorphan Reveal the Ent-Morphinan Pharmacophore of Tazopsine-Type Alkaloids. Pharmaceutics 2022, 14, 372. https: / /doi.org/10.3390/ pharmaceutics14020372

Academic Editor: Giuseppe De Rosa

Received: 29 December 2021 Accepted: 3 February 2022 Published: 7 February 2022

Publisher's Note: MDPI stays neutral with regard to jurisdictional claims in published maps and institutional affiliations.

Copyright: (C) 2022 by the authors. Licensee MDPI, Basel, Switzerland. This article is an open access article distributed under the terms and conditions of the Creative Commons Attribution (CC BY) license (https:// creativecommons.org/licenses/by/ $4.0 /)$.
1 UMR 261-MERIT, IRD, Université de Paris, 4 Avenue de l'Observatoire, 75006 Paris, France; antoinettekeita22@yahoo.com (A.K.); sandrine.houze@aphp.fr (S.H.)

2 Centre d'Immunologie et des Maladies Infectieuses, INSERM, CNRS, Sorbonne Université, 75013 Paris, France; jean-francois.franetich@upmc.fr (J.-F.F.); loise.valentin@gmail.com (L.V.); mallaurybordessoulles@gmail.com (M.B.); ludivine.baron.bio@gmail.com (L.B.); pierre.bigeard71@gmail.com (P.B.); maurel.tefit@upmc.fr (M.T.); dominique.mazier@sorbonne-universite.fr (D.M.); valerie.soulard@upmc.fr (V.S.)

3 UMR 152 Pharma-Dev, IRD, UPS, Université de Toulouse, 31400 Toulouse, France; maelle.carraz@ird.fr

4 Biopredic International, Parc d'Affaires de la Bretèche, Bldg A4, 35760 Saint-Grégoire, France

5 Laboratoire d'Excellence GR-Ex, 75015 Paris, France; florian.dupuy@inserm.fr (F.D.); catherine.lavazec@inserm.fr (C.L.)

6 Institut Cochin, Inserm U1016, CNRS UMR 8104, Université de Paris, 75014 Paris, France

7 UMR 8038-CiTCoM, CNRS, Université de Paris, 4 Avenue de l'Observatoire, 75006 Paris, France; valentine.geay@gmail.com (V.G.); sylvie.michel@u-paris.fr (S.M.)

8 CNR du Paludisme, AP-HP, Hôpital Bichat-Claude-Bernard, 46 Rue Henri-Huchard, 75018 Paris, France; veronique.sarrasin-hubert@aphp.fr

9 ISCR UMR CNRS 6226, Faculté de Pharmacie, Université de Rennes 1, 2 Avenue du Pr Léon Bernard, 35000 Rennes, France

* Correspondence: francois-hugues.poree@univ-rennes1.fr (F.-H.P.); romain.duval@ird.fr (R.D.)

+ These authors contributed equally to this work.

\begin{abstract}
The alkaloid tazopsine 1 was introduced in the late 2000s as a novel antiplasmodial hit compound active against Plasmodium falciparum hepatic stages, with the potential to develop prophylactic drugs based on this novel chemical scaffold. However, the structural determinants of tazopsine 1 bioactivity, together with the exact definition of the pharmacophore, remained elusive, impeding further development. We found that the antitussive drug dextromethorphan (DXM) 3, although lacking the complex pattern of stereospecific functionalization of the natural hit, was harboring significant antiplasmodial activity in vitro despite suboptimal prophylactic activity in a murine model of malaria, precluding its direct repurposing against the disease. The targeted $N$-alkylation of nor-DXM 15 produced a small library of analogues with greatly improved activity over DXM 3 against $P$. falciparum asexual stages. Amongst these, $N-2^{\prime}$-pyrrolylmethyl-nor-DXM 16i showed a 2- to 36-fold superior inhibitory potency compared to tazopsine $\mathbf{1}$ and DXM 3 against P. falciparum liver and blood stages, with respectively $760 \pm 130 \mathrm{nM}$ and $2.1 \pm 0.4 \mu \mathrm{M} \mathrm{IC}_{50}$ values, as well as liver/blood phase selectivity of 2.8. Furthermore, cpd. 16i showed a 5- to 8-fold increase in activity relative to DXM 3 against $P$. falciparum stages I-II and V gametocytes, with $18.5 \mu \mathrm{M}$ and $13.2 \mu \mathrm{M} \mathrm{IC}_{50}$ values, respectively. Cpd. 16i can thus be considered a promising novel hit compound against malaria in the ent-morphinan series with putative pan cycle activity, paving the way for further therapeutic development (e.g., investigation of its prophylactic activity in vivo).
\end{abstract}

Keywords: malaria; Plasmodium berghei; Plasmodium falciparum; hepatic stages; blood stages; prophylaxis; tazopsine; dextromethorphan; $N$-alkylation; hit compound 


\section{Introduction}

Malaria remains the major parasitic disease in the world, responsible for 229 million cases in 87 countries in 2019, associated with $>400,000$ deaths [1]. Malaria is also the most important infectious cause of mortality in children between 5 and 14 years old [2], principally from the deadliest and Africa-prevalent Plasmodium falciparum [3]. Malaria begins with the bite of a Plasmodium-infected female Anopheles mosquito, which injects sporozoites into the skin of the mammalian host. Sporozoites readily travel into the bloodstream, traverse several liver cells, and finally home into a hepatocyte. Once inside the host cell, sporozoites actively replicate and turn into multinucleated hepatic schizonts. At the end of the hepatic phase (i.e., 2-14 days after initial invasion, depending on the Plasmodium species), the schizonts and host cell rupture, releasing thousands of merozoites into the blood stream. Merozoites invade red blood cells, inside which they actively replicate, leading to erythrocytic schizonts that subsequently release more merozoites, which re-infect other erythrocytes in an exponential fashion. The symptomatology of malaria is directly associated with the parasite developmental phase in the blood, which is the principal target of most antimalarial drugs [4].

Semisynthetic artemisinin derivatives (ARTDs), associated with longer half-life companion drugs in artemisinin combination therapies (ACT), exert fast curative action against parasite blood stages and remain the frontline antimalarials prescribed worldwide. However, ARTDs are threatened by the rapid spread of artemisinin-resistant $P$. falciparum strains across Southeast Asia [5] and their independent emergence recently in Africa [6-8], which manifest a delayed clearance phenotype under conventional drug regimens [9]. This phenomenon is a worrying continuum of the history of chemoresistance by malaria parasites, which most exclusively affects drugs targeting the parasite blood phase. Indeed, the Plasmodium erythrocytic phase is characterized by important parasitemia and high mutations rates, allowing the selection of drug-resistant mutants $[4,10]$. On the other hand, the initial asymptomatic hepatic phase of parasite development features lower parasitemia and consequently lower mutation events, thus being considered an attractive target for malaria chemoprophylaxis. Drugs possessing novel chemical scaffolds active against parasite hepatic stages, either selectively [11] or as part of a pan-active mode of action [12,13], are therefore strongly pursued in drug discovery programs.

Tazopsine 1, an original ent-morphinan alkaloid isolated from the endemic Malagasy plant Strychnopsis thouarsii (Figure 1), induces low micromolar inhibition of P. falciparum liver and blood stages in vitro, but is insufficiently prophylactic at subtoxic doses in mice infected by P. yoelii ( $70 \%$ protection at $100 \mathrm{mg} / \mathrm{kg}$ ). On the other hand, the semisynthetic derivative $\mathrm{N}$-cyclopentyltazopsine 2 is 10 -fold less active, but 15 -fold more selective, than 1 towards liver stages in vitro. Furthermore, $\mathbf{2}$ is also less toxic than 1, enabling full prophylaxis at $200 \mathrm{mg} / \mathrm{kg}$ in the aforementioned malaria mouse model [14]. Despite patents filed in 2004 and 2006, these unprecedented antimalarial hits were not further investigated due to the difficult biosourcing of $\mathbf{1}$, its complex chemical structure from a total synthesis viewpoint, limited structure-activity relationships (SAR) [14,15], as well as the absence of identified pharmacophore and biological targets in the series. However, antiplasmodial properties are shared by other ent-morphinan alkaloids $[15,16]$, suggesting the existence of a common pharmacophore, irrespective of substitution and stereochemical patterns. Based on this rationale, we identified the generic antitussive drug dextromethorphan 3 (DXM 3, 3-methoxy-17-methylmorphinan) as possibly integrating the essential functional features of ent-morphinan alkaloids (Figure 1), having in mind its repurposing against malaria or use as a starting material towards simplified alkaloid mimics. The present paper describes the results of these endeavors. 


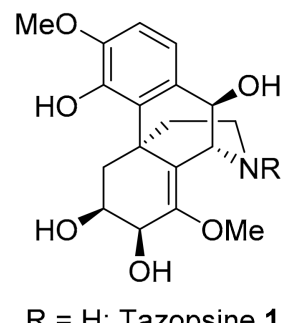

$\mathrm{R}=$ cyclopentyl: N-cyclopentyltazopsine $\mathbf{2}$

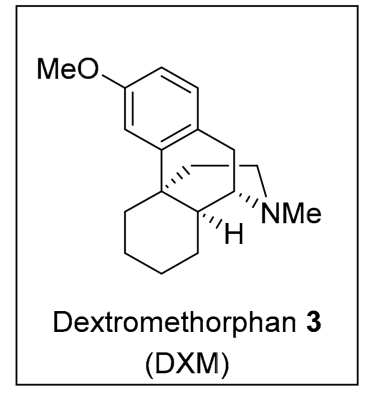

Figure 1. Antiplasmodial ent-morphinans: natural tazopsine 1, semisynthetic hit 2, and the prospected DXM 3.

\section{Experimental Section}

\subsection{Reagents, Solvents, and Equipment}

Reagents and anhydrous solvents were purchased from Sigma-Aldrich and were of the highest grade available. DXM 3 hydrobromide monohydrate, sinomenine 10, primaquine biphosphate (PQ), chloroquine biphosphate (CQ), and D-luciferin were purchased from Sigma-Aldrich. Tazopsine 1, 10-epi-tazopsine 4, sinococuline 5, and sinoacutine 9 were isolated from the plant Strychnopsis thouarsii as described previously [14,15]. DX 12 and Nor-DXM 15 were synthesized according to literature procedures [17,18]. Column chromatography was performed using silica gel 60 (9385 Merck). Thin-layer chromatography (TLC) was performed on aluminum plates coated with silica gel 60F254 (554 Merck), visualized with UV light (254 and $366 \mathrm{~nm})$, and revealed with sulfuric vanillin or phosphomolybdic acid reagents. NMR spectra $\left({ }^{1} \mathrm{H}\right.$ and $\left.{ }^{13} \mathrm{C}\right)$ were recorded on an Advance Bruker $400 \mathrm{MHz}$ spectrometer or an Oxford Instruments $600 \mathrm{MHz}$ spectrometer equipped with a BBI $600 \mathrm{MHz}$ probe, using solvent signal as an internal standard $\left(\mathrm{CDCl}_{3}: \delta\left({ }^{1} \mathrm{H}\right) 7.26\right.$ and $\delta$ $\left({ }^{13} \mathrm{C}\right) 77.16 \mathrm{ppm}, \mathrm{CD}_{3} \mathrm{OD}: \delta\left({ }^{1} \mathrm{H}\right) 3.31$ and $\left.\delta\left({ }^{13} \mathrm{C}\right) 49.00 \mathrm{ppm}\right)$. The $J$ coupling constants are provided in Hertz $(\mathrm{Hz})$. High-resolution mass spectra (HRMS) were recorded in the ESI mode on a LCT mass spectrometer (Waters) equipped with a TOF analyzer.

\subsection{Synthetic Chemistry}

2.2.1. Chemical Derivations of Tazopsine 1 (4,6,7,10-Tetrahydroxy-8,14-didehydro-3,8dimethoxymorphinan)

4-O-methyl-tazopsine $6, \mathrm{C}_{19} \mathbf{H}_{25} \mathrm{NO}_{6}$. A solution of tazopsine $\mathbf{1}$ free base $(20 \mathrm{mg}$, $0.057 \mathrm{mmol})$ in anhydrous $\mathrm{MeOH}(300 \mu \mathrm{L})$ was treated with diazomethane in excess at $0{ }^{\circ} \mathrm{C}$ for $12 \mathrm{~h}$. After removal of the solvent under reduced pressure, the residue was purified by silica gel column chromatography eluted with $\mathrm{CH}_{2} \mathrm{Cl}_{2} / \mathrm{MeOH} 0-10 v / v$ containing $1 \%$ of $20 \%$ aqueous $\mathrm{NH}_{3}$, yielding $6(8 \mathrm{mg}, 39 \%)$ as a white solid. ${ }^{1} \mathrm{H} \mathrm{NMR}(\mathrm{MeOD}, 400 \mathrm{MHz}): \delta$ $6.81(\mathrm{~d}, 1 \mathrm{H}, 8.4) ; 6.80(\mathrm{~d}, 1 \mathrm{H}, J=8.4) ; 4.45(\mathrm{~d}, 1 \mathrm{H}, J=2.1) ; 4.29(\mathrm{~d}, 1 \mathrm{H}, J=2.1) ; 4.27(\mathrm{~d}, 1 \mathrm{H}$, $J=2.6) ; 3.84(\mathrm{~m}, 1 \mathrm{H}) ; 3.62(\mathrm{~s}, 3 \mathrm{H}) ; 3.38(\mathrm{~s}, 3 \mathrm{H}) ; 3.28(\mathrm{~s}, 3 \mathrm{H}) ; 2.73(\mathrm{dd}, 1 \mathrm{H}, J=3.2,13.2) ; 2.62$ $(\mathrm{dd}, 1 \mathrm{H}, J=4.6,13.9) ; 2.36(\mathrm{ddd}, 1 \mathrm{H}, J=3.6,13.9,12.4) ; 2.08(\mathrm{dd}, 1 \mathrm{H}, J=13.2,13.2) ; 1.83$ $(\mathrm{dd}, 1 \mathrm{H}, J=3.6,12.3) ; 1.79$ (ddd, $1 \mathrm{H}, J=4.6,12.3,12.4)$. HRMS (ESI): $m / z$ calculated for $\mathrm{C}_{19} \mathrm{H}_{26} \mathrm{NO}_{6}{ }^{+}\left[\mathrm{M}+\mathrm{H}^{+}\right]=364.1760$. Found $=364.1752$.

General procedure for the reductive animation of tazopsine 1 (cpds. $7 \mathrm{a}-\mathrm{g}$ ). $\mathrm{N}$-alkyltazopsine derivatives were obtained from tazopsine using classical reductive amination of $37 \%$ aqueous formaldehyde or pure aldehydes by $\mathrm{NaBH}_{3} \mathrm{CN}$ [19]. Briefly, a stirred solution of tazopsine 1 free base $(34 \mathrm{mg}, 0.097 \mathrm{mmol})$ in anhydrous $\mathrm{MeOH}(600 \mu \mathrm{L})$ was primed by a gentle stream of argon for $15 \mathrm{~s}$. To this solution were added the aldehyde $(0.107 \mathrm{mmol})$ at r. t., followed after $10 \mathrm{~min}$ by $\mathrm{NaBH}_{3} \mathrm{CN}(95 \%, 6.4 \mathrm{mg}, 97 \mu \mathrm{mol})$. The mixture was stirred at $r$. $t$. under an argon atmosphere for $24 \mathrm{~h}$. After removal of the solvent under reduced pressure, the residue was slowly acidified with $1 \mathrm{M} \mathrm{HCl}$, then basified with $35 \%$ aqueous $\mathrm{NH}_{3}$, and evaporated under a vacuum. The residue was purified by silica gel column 
chromatography eluted with $\mathrm{CH}_{2} \mathrm{Cl}_{2} / \mathrm{MeOH}(0-10 v / v$ containing $1 \%$ of $20 \%$ aqueous $\mathrm{NH}_{3}$ ).

$N$-methyl-tazopsine $7 \mathrm{a}, \mathrm{C}_{19} \mathrm{H}_{25} \mathrm{NO}_{6}, 82 \%$ yield. ${ }^{1} \mathrm{H}$ NMR (MeOD, $\left.400 \mathrm{MHz}\right): \delta 6.90$ $(\mathrm{d}, 1 \mathrm{H}, J=8.4) ; 6.83(\mathrm{~d}, 1 \mathrm{H}, J=8.4) ; 4.70(\mathrm{~d}, 1 \mathrm{H}, J=2.2) ; 4.30(\mathrm{dd}, 1 \mathrm{H}, J=1.3,3.4) ; 4.21(\mathrm{~d}$, $1 \mathrm{H}, J=2.2) ; 3.88(\mathrm{~m}, 1 \mathrm{H}) ; 3.87(\mathrm{~s}, 3 \mathrm{H}) ; 3.70(\mathrm{~s}, 3 \mathrm{H}) ; 2.98(\mathrm{ddd}, 1 \mathrm{H}, J=1.3,4.1,13.8) ; 2.51(\mathrm{dd}$, $1 \mathrm{H}, J=3.3,12.4) ; 2.47(\mathrm{~s}, 3 \mathrm{H}) ; 2.26(\mathrm{~m}, 2 \mathrm{H}) ; 2.18(\mathrm{dd}, 1 \mathrm{H}, J=13.8,13.8) ; 1.95(\mathrm{~m}, 2 \mathrm{H})$. HRMS (ESI): $m / z$ calculated for $\mathrm{C}_{19} \mathrm{H}_{26} \mathrm{NO}_{6}{ }^{+}\left[\mathrm{M}+\mathrm{H}^{+}\right]=364.1760$. Found $=364.1764$.

$N$-n-propyl-tazopsine $7 \mathbf{b}, \mathbf{C}_{21} \mathbf{H}_{29} \mathrm{NO}_{6}, 68 \%$ yield. ${ }^{1} \mathrm{H}$ NMR (MeOD, $400 \mathrm{MHz}$ ): $\delta 6.99$ $(\mathrm{d}, 1 \mathrm{H}, J=8.4) ; 6.96(\mathrm{~d}, 1 \mathrm{H}, J=8.4) ; 4.87(\mathrm{~d}, 1 \mathrm{H}, J=1.9) ; 4.38(\mathrm{~d}, 1 \mathrm{H}, J=2.97) ; 3.92(\mathrm{~m}, 2 \mathrm{H})$; $3.88(\mathrm{~s}, 3 \mathrm{H}) ; 3.78(\mathrm{~s}, 3 \mathrm{H}) ; 3.09(\mathrm{~m}, 4 \mathrm{H}) ; 2.27(\mathrm{~d}, 1 \mathrm{H}, J=13.4) ; 2.14(\mathrm{dd}, 1 \mathrm{H}, J=4.0,12.3)$; $1.79(\mathrm{~m}, 4 \mathrm{H}) ; 1.04(\mathrm{t}, 3 \mathrm{H}, J=7.3)$. HRMS (ESI): $m / z$ calculated for $\mathrm{C}_{21} \mathrm{H}_{30} \mathrm{NO}_{6}{ }^{+}\left[\mathrm{M}+\mathrm{H}^{+}\right]=$ 392.2073. Found $=392.2064$.

$\mathrm{N}$-4'-hydroxybenzyl-tazopsine $7 \mathrm{c}^{\prime}, \mathrm{C}_{25} \mathrm{H}_{29} \mathrm{NO}_{7}, 45 \%$ yield. ${ }^{1} \mathrm{H}$ NMR (MeOD, $400 \mathrm{MHz}): \delta 7.35(\mathrm{~d}, 2 \mathrm{H}, J=8.6) ; 6.99(\mathrm{~d}, 1 \mathrm{H}, J=8.4) ; 6.92(\mathrm{~d}, 1 \mathrm{H}, J=8.4) ; 6.85(\mathrm{~d}, 2 \mathrm{H}$, $J=8.6) ; 4.41(\mathrm{~d}, 1 \mathrm{H}, J=3.3) ; 4.2(\mathrm{~m}, 2 \mathrm{H}) ; 3.98(\mathrm{ddd}, 1 \mathrm{H}, J=3.5,3.5,12.98) ; 3.88(\mathrm{~s}, 3 \mathrm{H}) ; 3.78$ $(\mathrm{m}, 4 \mathrm{H}) ; 3.05(\mathrm{~m}, 3 \mathrm{H}) ; 2.73(\mathrm{ddd}, 1 \mathrm{H}, J=4.1,13.0,13.0) ; 2.23(\mathrm{~m}, 3 \mathrm{H}) ; 2.08(\mathrm{dd}, 1 \mathrm{H}, J=2.3$, 13.7). HRMS (ESI): $m / z$ calculated for $\mathrm{C}_{25} \mathrm{H}_{30} \mathrm{NO}_{7}{ }^{+}\left[\mathrm{M}+\mathrm{H}^{+}\right]=456.2022$. Found $=456.2018$.

$\mathrm{N}$-4'-methoxybenzyl-tazopsine $7 \mathrm{~d}, \mathrm{C}_{26} \mathrm{H}_{31} \mathrm{NO}_{7}, 52 \%$ yield. ${ }^{1} \mathrm{H}$ NMR (MeOD, $400 \mathrm{MHz}): \delta 7.47(\mathrm{~d}, 2 \mathrm{H}, J=8.6) ; 7.0(\mathrm{~d}, 2 \mathrm{H}, J=8.6) ; 6.98(\mathrm{~d}, 1 \mathrm{H}, J=8.4) ; 6.93(\mathrm{~d}, 1 \mathrm{H}, J=8.4)$; $4.88(\mathrm{~d}, 1 \mathrm{H}, J=2.3) ; 4.71(\mathrm{~d}, 1 \mathrm{H}, J=2.3) ; 4.40(\mathrm{~d}, 1 \mathrm{H}, J=2.8) ; 4.25(\mathrm{~s}, 2 \mathrm{H}) ; 3.99(\mathrm{~m}, 1 \mathrm{H})$; $3.88(\mathrm{~s}, 3 \mathrm{H}) ; 3.83(\mathrm{~s}, 3 \mathrm{H}) ; 3.77(\mathrm{~s}, 3 \mathrm{H}) ; 3.06(\mathrm{~m}, 2 \mathrm{H}) ; 2.75(\mathrm{dd}, 1 \mathrm{H}, J=2.8,12.5) ; 2.28(\mathrm{ddd}$, $1 \mathrm{H}, J=3.2,13.6,13.6) ; 2.20(\mathrm{dd}, 1 \mathrm{H}, J=3.9,13.2) ; 2.07(\mathrm{dd}, 1 \mathrm{H}, J=2.1,13.2)$. HRMS (ESI): $m / z$ calculated for $\mathrm{C}_{26} \mathrm{H}_{32} \mathrm{NO}_{7}{ }^{+}\left[\mathrm{M}+\mathrm{H}^{+}\right]=470.2179$. Found $=470.2170$

$\mathrm{N}-3^{\prime}, 4^{\prime}$-methylenedioxybenzyl-tazopsine $7 \mathrm{e}, \mathrm{C}_{26} \mathrm{H}_{29} \mathrm{NO}_{8}, 52 \%$ yield. ${ }^{1} \mathrm{H}$ NMR (MeOD, $400 \mathrm{MHz}): \delta 7.91(\mathrm{~s}, 1 \mathrm{H}) ; 7.76(\mathrm{~d}, 1 \mathrm{H}, J=8.1) ; 7.54(\mathrm{~d}, 1 \mathrm{H}, J=8.1) ; 6.93(\mathrm{~d}, 1 \mathrm{H}$, $J=8.4) ; 6.88(\mathrm{~d}, 1 \mathrm{H}, J=8.4) ; 5.92(\mathrm{~s}, 2 \mathrm{H}) ; 4.78(\mathrm{~s}, 2 \mathrm{H}) ; 4.77(\mathrm{~d}, 1 \mathrm{H}, J=2.3) ; 4.56(\mathrm{~d}, 1 \mathrm{H}, J=2.3)$; $4.33(\mathrm{~d}, 1 \mathrm{H}, J=2.8) ; 3.91(\mathrm{~m}, 1 \mathrm{H}) ; 3.82(\mathrm{~s}, 3 \mathrm{H}) ; 3.75(\mathrm{~s}, 3 \mathrm{H}) ; 2.98(\mathrm{dd}, 1 \mathrm{H}, J=3.5,13.4) ; 2.65$ $(\mathrm{dd}, 1 \mathrm{H}, J=4.8,14.5) ; 2.45(\mathrm{~m}, 1 \mathrm{H}) ; 2.2(\mathrm{dd}, 1 \mathrm{H}, J=13.4,13.4) ; 2.15(\mathrm{dd}, 1 \mathrm{H}, J=3.5,12.5)$; $1.98(\mathrm{ddd}, 1 \mathrm{H}, J=4.8,12.5,12.5)$. HRMS (ESI): $m / z$ calculated for $\mathrm{C}_{26} \mathrm{H}_{30} \mathrm{NO}_{8}{ }^{+}\left[\mathrm{M}+\mathrm{H}^{+}\right]=$ 484.1971. Found $=484.1959$

$\mathrm{N}$-4'-chlorobenzyl-tazopsine $7 \mathrm{f}, \mathrm{C}_{25} \mathrm{H}_{28} \mathrm{NO}_{6} \mathrm{Cl}, 71 \%$ yield. ${ }^{1} \mathrm{H}$ NMR (MeOD, $400 \mathrm{MHz}): \delta 7.48(\mathrm{~d}, 2 \mathrm{H}, J=8.3) ; 7.32(\mathrm{~d}, 2 \mathrm{H}, J=8.3) ; 6.89(\mathrm{~d}, 1 \mathrm{H}, J=8.4) ; 6.82(\mathrm{~d}, 1 \mathrm{H}, J=6.4)$; $4.69(\mathrm{~s}, 2 \mathrm{H}) ; 4.65(\mathrm{~d}, 1 \mathrm{H}, J=2.1) ; 4.55(\mathrm{~d}, 1 \mathrm{H}, J=2.7) ; 4.43(\mathrm{~d}, 1 \mathrm{H}, J=2.1) ; 3.89(\mathrm{~m}, 1 \mathrm{H}) ; 3.84$ $(\mathrm{s}, 3 \mathrm{H}) ; 3.61(\mathrm{~s}, 3 \mathrm{H}) ; 2.96(\mathrm{dd}, 1 \mathrm{H}, J=3.2,13.8) ; 2.61(\mathrm{dd}, 1 \mathrm{H}, J=4.6,13.9) ; 2.39(\mathrm{ddd}, 1 \mathrm{H}$, $J=3.7,12.7,13.9) ; 2.15$ (dd, $1 \mathrm{H}, J=13.4,13.8) ; 2.02(\mathrm{dd}, 1 \mathrm{H}, J=3.7,12.7) ; 1.94$ (ddd, $1 \mathrm{H}$, $J=4.6,12.7,12.7$ ). HRMS (ESI): $m / z$ calculated for $\mathrm{C}_{25} \mathrm{H}_{29} \mathrm{NO}_{6} \mathrm{Cl}^{+}\left[\mathrm{M}+\mathrm{H}^{+}\right]=474.1683$. Found $=474.1678$.

$\mathrm{N}$-4'-bromobenzyl-tazopsine $7 \mathrm{~g}, \mathrm{C}_{25} \mathrm{H}_{28} \mathrm{NO}_{6} \mathrm{Br}, 61 \%$ yield. ${ }^{1} \mathrm{H}$ NMR (MeOD, $400 \mathrm{MHz}): \delta 7.51(\mathrm{~d}, 2 \mathrm{H}, J=8.4) ; 7.35(\mathrm{~d}, 2 \mathrm{H}, J=8.4) ; 6.92(\mathrm{~d}, 1 \mathrm{H}, J=8.4) ; 6.88(\mathrm{~d}, 1 \mathrm{H}, J=8.4) ;$ $4.78(\mathrm{~d}, 1 \mathrm{H}, J=2.2) ; 4.74(\mathrm{~s}, 2 \mathrm{H}) ; 4.73(\mathrm{~d}, 1 \mathrm{H}, J=2.2) ; 4.32(\mathrm{~d}, 1 \mathrm{H}, J=2.7) ; 3.93(\mathrm{ddd}, 1 \mathrm{H}$, $J=2.7,3.7,13.3) ; 3.88(\mathrm{~s}, 3 \mathrm{H}) ; 3.68(\mathrm{~s}, 3 \mathrm{H}) ; 3.0(\mathrm{dd}, 1 \mathrm{H}, J=2.9,13.9) ; 2.66(\mathrm{~m}, 1 \mathrm{H}) ; 2.46(\mathrm{ddd}$, $1 \mathrm{H}, J=3.9,12.1,12.1) ; 2.21(\mathrm{dd}, 1 \mathrm{H}, J=13.5,13.5) ; 2.05(\mathrm{ddd}, 1 \mathrm{H}, J=4.6,12.6,12.6) ; 1.96$ $(\mathrm{dd}, 1 \mathrm{H}, J=3.8,12.6)$. HRMS (ESI): $m / z$ calculated for $\mathrm{C}_{25} \mathrm{H}_{29} \mathrm{NO}_{6} \mathrm{Br}^{+}\left[\mathrm{M}+\mathrm{H}^{+}\right]=518.1178$. Found $=518.1166$.

$\mathrm{N}$-acetyl-tazopsine $8, \mathrm{C}_{20} \mathrm{H}_{25} \mathrm{NO}_{7}$. A solution of tazopsine $\mathbf{1}$ free base (34 $\mathrm{mg}, 0.097$ $\mathrm{mmol})$ in anhydrous $\mathrm{MeOH}(600 \mu \mathrm{L})$ was stirred at r. t. before adding $\mathrm{Ac}_{2} \mathrm{O}(0.097 \mathrm{mmol}$, $9.2 \mu \mathrm{L}$ ). The mixture was stirred at r. t. under an argon atmosphere for $1 \mathrm{~h}$. After removal of the solvent under reduced pressure, the residue was purified by silica gel column chromatography eluted with $\mathrm{CH}_{2} \mathrm{Cl}_{2} / \mathrm{MeOH}(0-5 \mathrm{v} / v)$, yielding $8(17 \mathrm{mg}, 45 \%)$ as a white solid. ${ }^{1} \mathrm{H}$ NMR (MeOD, $\left.400 \mathrm{MHz}\right)$ : first rotamer: $\delta 6.88(\mathrm{~d}, 1 \mathrm{H}, J=8.5) ; 6.81(\mathrm{~d}, 1 \mathrm{H}, J=8.5)$; $5.85(\mathrm{~d}, 1 \mathrm{H}, J=2.8) ; 4.39(\mathrm{~d}, 1 \mathrm{H}, J=2.8) ; 4.26(\mathrm{dd}, 1 \mathrm{H}, J=1.1,2.6) ; 3.68(\mathrm{dd}, 1 \mathrm{H}, J=5.8,12.9)$; $3.85(\mathrm{~s}, 3 \mathrm{H}) ; 3.81(\mathrm{~m}, 1 \mathrm{H}) ; 3.62(\mathrm{~s}, 3 \mathrm{H}) ; 3.03(\mathrm{ddd}, 1 \mathrm{H}, J=1.1,4.0,10.8) ; 2.78(\mathrm{ddd}, 1 \mathrm{H}, J=4.7$, $12.9,12.7) ; 2.23(\mathrm{dd}, 1 \mathrm{H}, J=5.5,10.8) ; 2.15(\mathrm{dd}, 1 \mathrm{H}, J=4.7,12.7) ; 2.0(\mathrm{~s}, 3 \mathrm{H}) ; 1.94(\mathrm{ddd}, 1 \mathrm{H}$, $J=5.8,12.7,12.7)$; second rotamer: $\delta 6.92(\mathrm{~d}, 1 \mathrm{H}, J=8.4) ; 6.83(\mathrm{~d}, 1 \mathrm{H}, J=8.4) ; 5.28(\mathrm{~d}, 1 \mathrm{H}$, 
$J=2.5) ; 4.49(\mathrm{~d}, 1 \mathrm{H}, J=2.5) ; 4.31(\mathrm{dd}, 1 \mathrm{H}, J=1.1,2.7) ; 4.12(\mathrm{dd}, 1 \mathrm{H}, J=5.7,13.9) ; 3.84(\mathrm{~s}, 3 \mathrm{H})$; $3.81(\mathrm{~m}, 1 \mathrm{H}) ; 3.74(\mathrm{~s}, 3 \mathrm{H}) ; 3.03(\mathrm{ddd}, 1 \mathrm{H}, J=1.1,4.0,10.8) ; 2.31$ (ddd, $1 \mathrm{H}, J=4.4,13.9,12.8$ ); $2.23(\mathrm{dd}, 1 \mathrm{H}, J=5.5,10.8) ; 2.21(\mathrm{~s}, 3 \mathrm{H}) ; 2.13(\mathrm{dd}, 1 \mathrm{H}, J=4.4,12.8) ; 1.78(\mathrm{ddd}, 1 \mathrm{H}, J=5.7,12.8$, 12.8). HRMS (ESI): $m / z$ calculated for $\mathrm{C}_{20} \mathrm{H}_{26} \mathrm{NO}^{+}\left[\mathrm{M}+\mathrm{H}^{+}\right]=392.1709$. Found $=392.1704$

\subsubsection{Chemical Derivations of DXM $[(9 \alpha, 13 \alpha, 14 \alpha)$-17-Methyl-3-methoxymorphinan 3}

DXM 3, $\mathrm{C}_{18} \mathrm{H}_{25} \mathrm{NO}$ (Generation of DXM 3 free base). A solution of $\mathrm{NaOH}(2.16 \mathrm{~g}$, $5.4 \mathrm{mmol}$ in $4 \mathrm{~mL}$ of $\mathrm{H}_{2} \mathrm{O}$ ) was added at $\mathrm{r}$. t. to a stirred suspension of dextromethorphan 3 hydrobromide monohydrate $(2 \mathrm{~g}, 5.4 \mathrm{mmol})$ in $8 \mathrm{~mL} \mathrm{CHCl}_{3}$, and the resulting mixture was stirred for $30 \mathrm{~min}$ at $\mathrm{r}$. t. The layers were separated in a separatory funnel and the organic phase was retrieved, dried over $\mathrm{MgSO}_{4}$, and filtered. The solvent was evaporated under reduced pressure to produce 3 free base as a dense and viscous off-white oil that solidified upon standing $(1.45 \mathrm{~g}, 5.3 \mathrm{mmol}, 99 \%$ yield). The analytical data were in accordance with the literature [18].

2-I-DXM 11, $(9 \alpha, 13 \alpha, 14 \alpha)$-2-iodo-17-methyl-3-methoxymorphinan, $\mathrm{C}_{18} \mathrm{H}_{24} \mathrm{INO}$. To a light-protected solution of DXM 3 free base $(21 \mathrm{mg}, 0.077 \mathrm{mmol})$ in $\mathrm{MeCN}(1 \mathrm{~mL})$ at $0{ }^{\circ} \mathrm{C}, \mathrm{N}$-iodosuccinimide $(20 \mathrm{mg}, 0.089 \mathrm{mmol})$ was added, followed by $p$-toluenesulfonic acid monohydrate $(27 \mathrm{mg}, 0.14 \mathrm{mmol})$. The mixture was allowed to warm to $\mathrm{r}$. $\mathrm{t}$. and was stirred overnight. The reaction mixture was treated with water $(1.5 \mathrm{~mL})$ and $1 \mathrm{M}$ $\mathrm{Na}_{2} \mathrm{~S}_{2} \mathrm{O}_{3}(1 \mathrm{~mL})$ and basified with a saturated solution of $\mathrm{Na}_{2} \mathrm{CO}_{3}$ to $\mathrm{pH} 10$. The aqueous phase was extracted with $\mathrm{CH}_{2} \mathrm{Cl}_{2}(4 \times 3 \mathrm{~mL})$, and the combined organic phases were dried over $\mathrm{Na}_{2} \mathrm{SO}_{4}$ and evaporated to dryness under reduced pressure. The crude product was purified by column chromatography on silica gel using $\mathrm{CH}_{2} \mathrm{Cl}_{2} / \mathrm{MeOH}$ (100:0 to 95:5 v/v containing $\left.1 \% \mathrm{NEt}_{3}\right)$ as eluent. 11 was obtained as a pale orange solid $(28 \mathrm{mg}, 0.070 \mathrm{mmol}$, 91\% yield). ${ }^{1} \mathrm{H}$ NMR $\left(\mathrm{CDCl}_{3}, 400 \mathrm{MHz}\right): \delta 7.50(\mathrm{~s}, 1 \mathrm{H}) ; 6.68(\mathrm{~s}, 1 \mathrm{H}) ; 3.83(\mathrm{~s}, 3 \mathrm{H}) ; 2.93$ $(\mathrm{d}, 1 \mathrm{H}, J=18.3) ; 2.82-2.80(\mathrm{~m}, 1 \mathrm{H}) ; 2.56(\mathrm{dd}, 1 \mathrm{H}, J=5.7,18.1) ; 2.48-2.45(\mathrm{~m}, 1 \mathrm{H}) ; 2.39(\mathrm{~s}$, $3 \mathrm{H}) ; 2.33-2.30(\mathrm{~m}, 1 \mathrm{H}) ; 2.06(\mathrm{td}, 1 \mathrm{H}, J=3.2,12.4) ; 1.87-1.83(\mathrm{~m}, 1 \mathrm{H}) ; 1.77(\mathrm{td}, 1 \mathrm{H}, J=4.8$, 12.7); $1.65-1.62(\mathrm{~m}, 2 \mathrm{H}) ; 1.42-1.25(\mathrm{~m}, 5 \mathrm{H}) ; 1.08(\mathrm{qd}, 1 \mathrm{H}, J=3.7,12.5) .{ }^{13} \mathrm{C} \mathrm{NMR}\left(\mathrm{CDCl}_{3}\right.$, $100 \mathrm{MHz}): \delta 157.0,142.2,138.5,132.2,108.2,83.1,58.0,56.6,47.3,45.1,42.8,41.8,37.5,36.7$, $26.8,26.5,23.2,22.3$. HRMS (ESI): $m / z$ calculated for $\mathrm{C}_{18} \mathrm{H}_{25} \mathrm{NOI}^{+}\left[\mathrm{M}+\mathrm{H}^{+}\right]=398.0975$. Found $=398.0973$. The analytical data were in accordance with the literature [17].

DX 12, $(9 \alpha, 13 \alpha, 14 \alpha)$-17-methyl-3-hydroxymorphinan, $\mathrm{C}_{17} \mathrm{H}_{23} \mathrm{NO}$ (O-Demethylation of DXM 3). To a solution of aqueous $\mathrm{HBr}(48 \%, 8 \mathrm{~mL})$, dextromethorphan 3 hydrobromide monohydrate $(1.61 \mathrm{~g}, 4.35 \mathrm{mmol})$ was stirred in, and the resulting solution was heated to reflux overnight. The reaction mixture was cooled in an ice bath and basified to $\mathrm{pH} 8$ by $2 \mathrm{~N} \mathrm{NaOH}$ aqueous solution, then extracted with $\mathrm{CHCl}_{3}(5 \times 20 \mathrm{~mL})$. The combined organic phases were dried over $\mathrm{Na}_{2} \mathrm{SO}_{4}$ and evaporated under a vacuum to produce DX 12 as a white solid (1.02 g, $3.96 \mathrm{mmol}, 91 \%$ yield). An analytical sample was purified by column chromatography on silica gel using $\mathrm{CH}_{2} \mathrm{Cl}_{2} / \mathrm{MeOH}$ (98:2 to $97: 3 v / v$ containing $1 \%$ $\left.\mathrm{NEt}_{3}\right)$ as eluent. ${ }^{1} \mathrm{H}$ NMR $\left(\mathrm{CDCl}_{3}, 300 \mathrm{MHz}\right): \delta 6.95(\mathrm{~d}, 1 \mathrm{H}, J=8.2) ; 6.70(\mathrm{~d}, 1 \mathrm{H}, J=2.6) ; 6.61$ $(\mathrm{dd}, 1 \mathrm{H}, J=2.6,8.2) ; 2.99(\mathrm{~d}, 1 \mathrm{H}, J=18.2) ; 2.91-2.86(\mathrm{~m}, 1 \mathrm{H}) ; 2.66(\mathrm{dd}, 1 \mathrm{H}, J=5.8,18.2)$; 2.56-2.47 (m, 1H); $2.41(\mathrm{~s}, 3 \mathrm{H}) ; 2.87-2.16(\mathrm{~m}, 2 \mathrm{H}) ; 1.94-1.86(\mathrm{~m}, 1 \mathrm{H}) ; 1.77(\mathrm{td}, 1 \mathrm{H}, J=4.2$, $12.7) ; 1.65-1.56(\mathrm{~m}, 1 \mathrm{H}) ; 1.46-1.25(\mathrm{~m}, 7 \mathrm{H}) ; 1.23-1.06(\mathrm{~m}, 1 \mathrm{H})$. The analytical data were in accordance with the literature [17].

2-I-DX 13, $(9 \alpha, 13 \alpha, 14 \alpha)$-2-iodo-17-methyl-3-hydroxymorphinan, $\mathrm{C}_{17} \mathrm{H}_{22} \mathrm{INO}$. To a light-protected solution of $\mathbf{1 2}(21 \mathrm{mg}, 0.082 \mathrm{mmol})$ in $\mathrm{MeCN}(1.5 \mathrm{~mL})$ at $0{ }^{\circ} \mathrm{C}, \mathrm{N}$ iodosuccinimide $(20 \mathrm{mg}, 0.089 \mathrm{mmol})$ was added, followed by $p$-toluenesulfonic acid monohydrate $(27 \mathrm{mg}, 0.14 \mathrm{mmol})$. The mixture was allowed to warm up to $\mathrm{r}$. $\mathrm{t}$. and was stirred overnight. The reaction mixture was treated with water $(1.5 \mathrm{~mL})$ and $1 \mathrm{M} \mathrm{Na}_{2} \mathrm{~S}_{2} \mathrm{O}_{3}$ $(1.5 \mathrm{~mL})$, and basified with a solution of saturated $\mathrm{Na}_{2} \mathrm{CO}_{3}$ to $\mathrm{pH} 10$. The aqueous phase was extracted with $\mathrm{CH}_{2} \mathrm{Cl}_{2}(4 \times 1 \mathrm{~mL})$, and the combined organic phases were dried over $\mathrm{Na}_{2} \mathrm{SO}_{4}$ and evaporated to dryness under reduced pressure. The crude product was purified by column chromatography on silica gel using $\mathrm{CH}_{2} \mathrm{Cl}_{2} / \mathrm{MeOH}$ (100:0 to 95:5 v/v containing $\left.1 \% \mathrm{NEt}_{3}\right)$ as eluent to afford $13(27 \mathrm{mg}, 87 \%) .{ }^{1} \mathrm{H} \mathrm{NMR}\left(\mathrm{CDCl}_{3}, 400 \mathrm{MHz}\right): \delta 7.42$ 
$(\mathrm{s}, 1 \mathrm{H}) ; 6.78(\mathrm{~s}, 1 \mathrm{H}) ; 5.29(\mathrm{ls}, 1 \mathrm{H}) ; 2.95-2.91(\mathrm{~m}, 2 \mathrm{H}) ; 2.66(\mathrm{dd}, 1 \mathrm{H}, J=5.9,18.6) ; 2.59-2.55(\mathrm{~m}$, $1 \mathrm{H}) ; 2.44(\mathrm{~s}, 3 \mathrm{H}) ; 2.22-2.15(\mathrm{~m}, 2 \mathrm{H}) ; 1.91-1.88(\mathrm{~m}, 1 \mathrm{H}) ; 1.77(\mathrm{td}, 1 \mathrm{H}, J=4.7,12.9) ; 1.62-1.59$ $(\mathrm{m}, 1 \mathrm{H}) ; 1.47-1.38(\mathrm{~m}, 2 \mathrm{H}) ; 1.33-1.18(\mathrm{~m}, 4 \mathrm{H}) ; 1.07(\mathrm{qd}, 1 \mathrm{H}, J=3.7,12.4)$. The analytical data were in accordance with the literature [17].

$(9 \alpha, 13 \alpha, 14 \alpha)-2^{\prime}, 2^{\prime}, 2^{\prime}$-trichloroethyl-17-carboxylate-3-methoxymorphinan $14, \mathrm{C}_{20} \mathrm{H}_{24}$ $\mathrm{NO}_{3} \mathrm{Cl}_{3}$ ( $\mathrm{N}$-demethylation of DXM 3, step 1$)$. To a solution of DXM 3 free base (1.3 $\mathrm{g}$, $4.8 \mathrm{mmol})$ in toluene $(3 \mathrm{~mL}), 2^{\prime}, 2^{\prime}, 2^{\prime}$-trichloroethylchloroformate $(800 \mu \mathrm{L}, 5.8 \mathrm{mmol})$ was added, and the reaction mixture was heated under reflux for $2 \mathrm{~h}$. The mixture was cooled down to $\mathrm{r}$. $\mathrm{t}$., then washed with $5 \% \mathrm{HCl}(3 \mathrm{~mL})$, then with water $(3 \mathrm{~mL})$. The organic layer was separated, dried over anhydrous $\mathrm{Na}_{2} \mathrm{SO}_{4}$, then evaporated under reduced pressure. The crude product was purified by column chromatography on silica gel using EtOAc/cyclohexane $(10: 90 \mathrm{v} / \mathrm{v})$ as eluent to produce $14(3.1 \mathrm{~g}, 92 \%)$ as a white solid. ${ }^{1} \mathrm{H}$ $\mathrm{NMR}\left(\mathrm{CDCl}_{3}, 400 \mathrm{MHz}\right)$ : Amide rotamers [7.03 and $\left.7.00(\mathrm{~d}, 1 \mathrm{H}, J=8.4)\right] ; 6.83(\mathrm{~d}, 1 \mathrm{H}$, $J=2.4) ; 6.73(\mathrm{dd}, 1 \mathrm{H}, J=2.4,8.4) ; 4.85(\mathrm{t}, 1 \mathrm{H}, J=6.5) ; 4.75(\mathrm{q}, 1 \mathrm{H}, J=12.0) ; 4.39(\mathrm{t}, 1 \mathrm{H}$, $J=4.4) ; 3.92(\mathrm{td}, 1 \mathrm{H}, J=4.5,12.8) ; 3.79(\mathrm{~s}, 3 \mathrm{H}) ; 3.13(\mathrm{dd}, 1 \mathrm{H}, J=6.0,18.1) ; 2.79-2.26(\mathrm{~m}, 2 \mathrm{H})$; $2.38(\mathrm{~m}, 1 \mathrm{H}) ; 1.74-1.26(\mathrm{~m}, 9 \mathrm{H}) ; 1.09(\mathrm{~m}, 1 \mathrm{H}) .{ }^{13} \mathrm{C}\left(\mathrm{CDCl}_{3}, 100 \mathrm{MHz}\right): \delta 158.6,153.7,140.2$, $129.1,128.1,111.4,96.0,95.1,55.3,50.7,44.0,41.7,38.9,37.6,36.5,31.4,26.6,26.4,22.1$. The analytical data were in accordance with the literature $[18,20]$.

Nor-DXM 15, $(9 \alpha, 13 \alpha, 14 \alpha)$-3-hydroxymorphinan, $\mathrm{C}_{17} \mathrm{H}_{23} \mathrm{NO}(\mathrm{N}$-demethylation of DXM 3, step 2). To a solution of $(9 \alpha, 13 \alpha, 14 \alpha)-2^{\prime}, 2^{\prime}, 2^{\prime}$-trichloroethyl-17-carboxylate-3methoxy morphinan 14 ( $3 \mathrm{~g}, 6.93 \mathrm{mmol})$ in aqueous $\mathrm{AcOH}(90 \%, 30 \mathrm{~mL})$, zinc powder $(1.36$ $\mathrm{g}$, $20.8 \mathrm{mmol}$ ) was added in several portions over $30 \mathrm{~min}$. After $1 \mathrm{~h}$ of additional stirring, the zinc powder was filtered off using celite ${ }^{\circledR}$, and the solvent evaporated under reduced pressure. Toluene $(6 \mathrm{~mL})$ was added to solubilize the obtained dense oil, the mixture was brought to reflux, then allowed to cool down to $4{ }^{\circ} \mathrm{C}$. The resulting white precipitate of $(9 \alpha, 13 \alpha, 14 \alpha)$-3-methoxymorphinan tetraacetozincate was filtered off and washed four times with $\mathrm{Et}_{2} \mathrm{O}(5 \mathrm{~mL})$. The precipitate was then dissolved in $\mathrm{CHCl}_{3}(6 \mathrm{~mL})$ and basified to $\mathrm{pH} 9.5$ with $1 \mathrm{M} \mathrm{NaOH}$ aqueous solution. The obtained white suspension was shaken with $\mathrm{CHCl}_{3}(30 \mathrm{~mL})$, filtered using fritted glass, and the organic layer was separated. The aqueous layer was further extracted with $\mathrm{CHCl}_{3}(4 \times 10 \mathrm{~mL})$, the organic layers were combined, dried over anhydrous $\mathrm{Na}_{2} \mathrm{SO}_{4}$, and the solvent was evaporated under reduced pressure to produce nor-DXM $15(0.832 \mathrm{~g}, 3.23 \mathrm{mmol}, 47 \%$ yield $)$ as a clear oil that solidified upon standing. ${ }^{1} \mathrm{H} \mathrm{NMR}\left(\mathrm{CDCl}_{3}, 400 \mathrm{MHz}\right): \delta 9.55(\mathrm{ls}, 1 \mathrm{H}) ; 6.98(\mathrm{~d}, 1 \mathrm{H}, J=8.4) ; 6.69(\mathrm{~d}, 1 \mathrm{H}$, $J=2.6) ; 6.35(\mathrm{dd}, 1 \mathrm{H}, J=2.6,8.4) ; 3.67(\mathrm{~s}, 3 \mathrm{H}) ; 3.63-3.58(\mathrm{~m}, 1 \mathrm{H}) ; 3.19-3.03(\mathrm{~m}, 3 \mathrm{H}) ; 2.63(\mathrm{t}$, $J=12.0,1 \mathrm{H}) ; 2.24(\mathrm{~d}, 1 \mathrm{H}, J=13.6) ; 2.08(\mathrm{~d}, 1 \mathrm{H}, J=12.0) ; 1.92(\mathrm{td}, 1 \mathrm{H}, J=43.6,13.6) ; 1.53(\mathrm{~d}$, $1 \mathrm{H}, J=12.4) ; 1.46-1.25(\mathrm{~m}, 5 \mathrm{H}) ; 1.21-1.12(\mathrm{~m}, 1 \mathrm{H}) ; 0.96(\mathrm{qd}, 1 \mathrm{H}, J=2.4,12.4)$. The analytical data were in accordance with the literature $[18,20]$.

General procedure for the reductive animation of nor-DXM 15 (cpds. 16a-m). To a stirred solution of nor-DXM $15(30 \mathrm{mg}, 0.117 \mathrm{mmol})$ in anhydrous DMF $(300 \mu \mathrm{L})$, the corresponding aldehyde $(0.129 \mathrm{mmol})$ was added at r. t. under an argon atmosphere. After $10 \mathrm{~min}, \mathrm{STABH}(97 \%, 51 \mathrm{mg}, 234 \mu \mathrm{mol})$ was added in one portion. The resulting suspension was stirred until completion (TLC monitoring, Table 1), then, $\mathrm{H}_{2} \mathrm{O}(100 \mu \mathrm{L})$ was added and the reaction mixture was partitioned in a system composed of EtOAc $(300 \mu \mathrm{L})$ and $\mathrm{NaHCO}_{3} / \mathrm{Na}_{2} \mathrm{CO}_{3}$ buffer $(\mathrm{pH} 9.5,300 \mu \mathrm{L})$. After separation, the organic phase was washed with carbonate buffer $(3 \times 300 \mu \mathrm{L})$, dried over $\mathrm{Na}_{2} \mathrm{SO}_{4}$, and concentrated under reduced pressure. The crude product was purified by column chromatography on silica gel using cyclohexane/EtOAc (95:5 to 90:10 $v / v$ containing 1\% $\mathrm{NEt}_{3}$ ) as eluent, yielding $N$-substituted $(9 \alpha, 13 \alpha, 14 \alpha)$-3-methoxymorphinans $\mathbf{1 6 a - m}$ at 33-97\%. 
Table 1. Reaction times to synthesize cpds. $\mathbf{1 6 a}-\mathbf{1 6 m}$ by reductive amination with STABH, and their yields.

\begin{tabular}{ccc}
\hline Cpd. & Reaction Time (h) & Yield (\%) \\
\hline $\mathbf{1 6 a}$ & 2 & 45 \\
\hline $\mathbf{1 6 b}$ & 2 & 75 \\
\hline $\mathbf{1 6 c}$ & 2 & 56 \\
\hline $\mathbf{1 6 d}$ & 2 & 56 \\
\hline $\mathbf{1 6 e}$ & 2 & 52 \\
\hline $\mathbf{1 6 f}$ & 2 & 68 \\
\hline $\mathbf{1 6 g}$ & 2 & 35 \\
\hline $\mathbf{1 6 h}$ & 2 & 68 \\
\hline $\mathbf{1 6 i}$ & 2 & 53 \\
\hline $\mathbf{1 6 j}$ & 21 & 48 \\
\hline $\mathbf{1 6 k}$ & 15 & 33 \\
\hline $\mathbf{1 6 1}$ & 2 & 82 \\
\hline $\mathbf{1 6 m}$ & 2 & 97 \\
\hline
\end{tabular}

Cpd. 16a, $(9 \alpha, 13 \alpha, 14 \alpha)-17-n$-propyl-3-methoxymorphinan, $\mathrm{C}_{20} \mathrm{H}_{29} \mathrm{NO} .{ }^{1} \mathrm{H}$ NMR $\left(\mathrm{CDCl}_{3}, 600 \mathrm{MHz}\right): \delta 7.02(\mathrm{~d}, 1 \mathrm{H}, J=8.3) ; 6.80(\mathrm{~d}, 1 \mathrm{H}, J=2.4) ; 6.69(\mathrm{dd}, 1 \mathrm{H}, J=2.4$, 8.3); 3.79 (s, 3H); 3.78-3.95 (m, 2H); $2.61(\mathrm{dd}, 1 \mathrm{H}, J=4.9,17.9) ; 2.55(\mathrm{~d}, 1 \mathrm{H}, J=9.5) ; 2.51-2.45$ $(\mathrm{m}, 2 \mathrm{H}) ; 2.34(\mathrm{~d}, 1 \mathrm{H}, J=13.3) ; 2.06(\mathrm{t}, 1 \mathrm{H}, J=11) ; 1.86(\mathrm{~d}, 1 \mathrm{H}, J=10) ; 1.77(\mathrm{t}, 1 \mathrm{H}, J=10.7) ; 1.63$ $(\mathrm{d}, 1 \mathrm{H}, J=12.1) ; 1.55-1.50(\mathrm{~m}, 3 \mathrm{H}) ; 1.42-1.25(\mathrm{~m}, 5 \mathrm{H}) ; 1.13(\mathrm{qd}, 1 \mathrm{H}, \mathrm{J}=3.5,12.6) ; 0.93-0.90(\mathrm{t}$, $3 \mathrm{H}, J=7.2) .{ }^{13} \mathrm{C} \mathrm{NMR}\left(\mathrm{CDCl}_{3}, 150 \mathrm{MHz}\right): \delta 158.3,141.9,130.0,128.6,111.2,110.8,57.2,56.1$, 55.3, 46.0, 45.0, 42.0, 38.0, 36.7, 27.0, 26.7, 24.1, 22.4, 20.9, 12.2. HRMS (ESI): $m / z$ calculated for $\mathrm{C}_{20} \mathrm{H}_{30} \mathrm{NO}^{+}[\mathrm{M}+\mathrm{H}]^{+}=300.2322$. Found $=300.2323$.

Cpd. $16 \mathrm{~b},(9 \alpha, 13 \alpha, 14 \alpha)-17-n$-butyl-3-methoxymorphinan, $\mathrm{C}_{21} \mathrm{H}_{31} \mathrm{NO}$. ${ }^{1} \mathrm{H} \mathrm{NMR}$ $\left(\mathrm{CDCl}_{3}, 300 \mathrm{MHz}\right): \delta 7.02(\mathrm{~d}, 1 \mathrm{H}, J=9.0) ; 6.80(\mathrm{~d}, 1 \mathrm{H}, J=3.0) ; 6.69(\mathrm{dd}, 1 \mathrm{H}, J=3.0,9.0) ; 3.78$ $(\mathrm{s}, 3 \mathrm{H}) ; 2.96-2.90(\mathrm{~m}, 2 \mathrm{H}) ; 2.64-2.45(\mathrm{~m}, 4 \mathrm{H}) ; 2.35-2.32(\mathrm{~m}, 1 \mathrm{H}) ; 2.08-2.00(\mathrm{td}, 1 \mathrm{H}, J=3.0,9.0)$; $1.87-1.72(\mathrm{~m}, 2 \mathrm{H}) ; 1.64-1.61(\mathrm{~m}, 1 \mathrm{H}) ; 1.54-1.45(\mathrm{~m}, 3 \mathrm{H}) ; 1.44-1.27(\mathrm{~m}, 7 \mathrm{H}) ; 1.13-1.08(\mathrm{~m}$, $1 \mathrm{H}) ; 0.94-0.90(\mathrm{t}, 3 \mathrm{H}, J=6.0) .{ }^{13} \mathrm{C} \mathrm{NMR}\left(\mathrm{CDCl}_{3}, 75 \mathrm{MHz}\right): \delta 158.2,141.8,129.8,128.5,111.1$, $110.7,55.8,55.2,54.8,45.9,45.0,41.9,37.9,36.6,29.8,26.9,26.6,23.9,22.3,21.0,14.2$. HRMS (ESI): $m / z$ calculated for $\mathrm{C}_{21} \mathrm{H}_{32} \mathrm{NO}^{+}[\mathrm{M}+\mathrm{H}]^{+}=314.2478$. Found $=314.2480$.

Cpd. 16c, $(9 \alpha, 13 \alpha, 14 \alpha)-17-n$-pentyl-3-methoxymorphinan, $\mathrm{C}_{22} \mathrm{H}_{33} \mathrm{NO} .{ }^{1} \mathrm{H} \mathrm{NMR}$ $\left(\mathrm{CDCl}_{3}, 400 \mathrm{MHz}\right): \delta 7.00(\mathrm{~d}, 1 \mathrm{H}, J=8.3) ; 6.80(\mathrm{~d}, 1 \mathrm{H}, J=2.6) ; 6.69(\mathrm{dd}, 1 \mathrm{H}, J=2.6,8.3)$; $3.78(\mathrm{~s}, 3 \mathrm{H}) ; 2.96-2.89(\mathrm{~m}, 2 \mathrm{H}) ; 2.61(\mathrm{~d}, 1 \mathrm{H}, J=5.5) ; 2.56-2.44(\mathrm{~m}, 3 \mathrm{H}) ; 2.33(\mathrm{~d}, 1 \mathrm{H}, J=12.3)$; $2.04-2.01(\mathrm{~m}, 1 \mathrm{H}) ; 1.84(\mathrm{~d}, 1 \mathrm{H}, J=12.4) ; 1.76(\mathrm{~m}, 1 \mathrm{H}) ; 1.63(\mathrm{~m}, 1 \mathrm{H}) ; 1.54-1.47(\mathrm{~m}, 3 \mathrm{H})$; $1.41-1.26(\mathrm{~m}, 9 \mathrm{H}) ; 1.13-1.09(\mathrm{qd}, 1 \mathrm{H} J=2.8,11.8) ; 0.89(\mathrm{t}, 3 \mathrm{H}, J=6.8) .{ }^{13} \mathrm{C} \mathrm{NMR}\left(\mathrm{CDCl}_{3}\right.$, $100 \mathrm{MHz}$ ): $\delta$ 158.3, 142.0, 130.0, 128.6, 111.2, 110.8, 56.0, 55.3, 55.2, 46.0, 45.2, 42.0, 38.0, 36.8, $30.1,27.5,27.0,26.7,24.0,22.8,22.4,14.2$. HRMS (ESI): $m / z$ calculated for $\mathrm{C}_{22} \mathrm{H}_{34} \mathrm{NO}^{+}[\mathrm{M}+$ $\mathrm{H}]^{+}=328.2635$. Found $=328.2635$.

Cpd. 16d, $(9 \alpha, 13 \alpha, 14 \alpha)$-17-cyclopropylmethyl-3-methoxymorphinan $\mathrm{C}_{21} \mathrm{H}_{29} \mathrm{NO}$. ${ }^{1} \mathrm{H} \mathrm{NMR}\left(\mathrm{CDCl}_{3}, 400 \mathrm{MHz}\right): \delta 7.00(\mathrm{~d}, 1 \mathrm{H}, J=8.4) ; 6.80(\mathrm{~d}, 1 \mathrm{H}, J=2.5) ; 6.69(\mathrm{dd}, 1 \mathrm{H}, J=2.5$, $8.4) ; 3.80(\mathrm{~s}, 3 \mathrm{H}) ; 3.17-3.12(\mathrm{~m}, 1 \mathrm{H}) ; 2.88(\mathrm{~d}, 1 \mathrm{H}, J=18.2) ; 2.77-2.71(\mathrm{~m}, 1 \mathrm{H}) ; 2.65-2.60(\mathrm{~m}$, $1 \mathrm{H}) ; 2.55-2.50(\mathrm{~m}, 1 \mathrm{H}) ; 2.35(\mathrm{~d}, 2 \mathrm{H}, J=13.0) ; 2.03(\mathrm{t}, 1 \mathrm{H}, J=13.2) ; 1.91(\mathrm{~d}, 1 \mathrm{H}, J=9.6) ; 1.82$ $(\mathrm{t}, 1 \mathrm{H}, J=11.5) ; 1.64(\mathrm{~d}, 1 \mathrm{H}, J=13.0) ; 1.55-1.49(\mathrm{~m}, 1 \mathrm{H}) ; 1.44-1.27(\mathrm{~m}, 5 \mathrm{H}) ; 1.15(\mathrm{qd}, 1 \mathrm{H}$, $J=2.4,12.4) ; 0.95-0.87(\mathrm{~m}, 1 \mathrm{H}) ; 0.55-0.50(\mathrm{~m}, 2 \mathrm{H}) ; 0.16-0.12(\mathrm{~m}, 2 \mathrm{H}) .{ }^{13} \mathrm{C} \mathrm{NMR}\left(\mathrm{CDCl}_{3}\right.$, $100 \mathrm{MHz}$ ): $\delta$ 158.6, 142.1, 130.1, 128.8, 111.4, 111.1, 60.2, 56.3, 55.5, 46.2, 45.3, 42.2, 38.2, 36.9, 27.3, 26.9, 24.3, 22.6, 4.5, 4.01. HRMS (ESI): $m / z$ calculated for $\mathrm{C}_{21} \mathrm{H}_{30} \mathrm{NO}^{+}[\mathrm{M}+\mathrm{H}]^{+}=$ 312.2322. Found $=312.2341$. 
Cpd. 16e, $(9 \alpha, 13 \alpha, 14 \alpha)$-17-cyclopentylmethyl-3-methoxy morphinan $\mathrm{C}_{23} \mathrm{H}_{33} \mathrm{NO}$. ${ }^{1} \mathrm{H} \mathrm{NMR}\left(\mathrm{CDCl}_{3}, 600 \mathrm{MHz}\right): \delta 7.00(\mathrm{~d}, 1 \mathrm{H}, J=8.3) ; 6.80(\mathrm{~d}, 1 \mathrm{H}, J=2.5) ; 6.69(\mathrm{dd}, 1 \mathrm{H}, J=2.2$, 8.3); $3.78(\mathrm{~s}, 3 \mathrm{H}) ; 2.92(\mathrm{~d}, 1 \mathrm{H}, J=17.7) ; 2.66-2.37(\mathrm{~m}, 1 \mathrm{H}) ; 2.33(\mathrm{~d}, 1 \mathrm{H}, J=13.3) ; 2.13-2.00$ $(\mathrm{m}, 2 \mathrm{H}) ; 1.86-1.71(\mathrm{~m}, 4 \mathrm{H}) ; 1.66-1.56(\mathrm{~m}, 4 \mathrm{H}) ; 1.54-1.50(\mathrm{~m}, 4 \mathrm{H}) ; 1.42-1.20(\mathrm{~m}, 8 \mathrm{H}) ; 1.10$ (qd, $1 \mathrm{H}, J=3.9,12.5$ ). HRMS (ESI): $m / z$ calculated for $\mathrm{C}_{21} \mathrm{H}_{30} \mathrm{NO}^{+}[\mathrm{M}+\mathrm{H}]^{+}=340.2635$. Found $=340.2636$.

Cpd. 16f, $(9 \alpha, 13 \alpha, 14 \alpha)$-17-cyclohexylmethyl-3-methoxy morphinan, $\mathrm{C}_{24} \mathrm{H}_{35} \mathrm{NO} .{ }^{1} \mathrm{H}$ NMR $\left(\mathrm{CDCl}_{3}, 600 \mathrm{MHz}\right): \delta 7.00(\mathrm{~d}, 1 \mathrm{H}, J=8.3) ; 6.80(\mathrm{~d}, 1 \mathrm{H}, J=2.3) ; 6.68(\mathrm{dd}, 1 \mathrm{H}, J=2.3$, $8.3) ; 3.80(\mathrm{~s}, 3 \mathrm{H}) ; 2.91(\mathrm{~d}, 1 \mathrm{H}, J=17.9) ; 2.79-2.75(\mathrm{~m}, 1 \mathrm{H}) ; 2.58(\mathrm{~d}, 1 \mathrm{H}, J=17.0) ; 2.45-2.40(\mathrm{~m}$, $1 \mathrm{H}) ; 2.34-2.25(\mathrm{~m}, 3 \mathrm{H}) ; 2.07-2.01(\mathrm{~m}, 1 \mathrm{H}) ; 1.81(\mathrm{~d}, 1 \mathrm{H}, J=12.2) ; 1.75-1.62(\mathrm{~m}, 6 \mathrm{H}) ; 1.50(\mathrm{~d}$, $1 \mathrm{H}, J=11.3) ; 1.40-1.33(\mathrm{~m}, 11 \mathrm{H}) ; 0.93-0.82(\mathrm{~m}, 2 \mathrm{H}) .{ }^{13} \mathrm{C} \mathrm{NMR}\left(\mathrm{CDCl}_{3}, 150 \mathrm{MHz}\right): \delta 158.2$, 142.2, 130.0, 128.7, 111.2, 110.7, 62.3, 56.7, 55.3, 46.3, 45.3, 42.3, 38.4, 36.8, 35.9, 32.2, 27.1, 27.0, 26.8, 26.4, 24.6, 22.4. HRMS (ESI): $m / z$ calculated for $\mathrm{C}_{24} \mathrm{H}_{36} \mathrm{NO}^{+}[\mathrm{M}+\mathrm{H}]^{+}=354.2791$. Found $=354.2790$.

Cpd. $16 \mathrm{~g},(9 \alpha, 13 \alpha, 14 \alpha)$-17-(2'-furanylmethyl)-3-methoxy morphinan, $\mathrm{C}_{22} \mathrm{H}_{27} \mathrm{NO}_{2}$. ${ }^{1} \mathrm{H} \mathrm{NMR}\left(\mathrm{CDCl}_{3}, 400 \mathrm{MHz}\right): \delta 7.39-7.38(\mathrm{~m}, 1 \mathrm{H}) ; 7.04(\mathrm{~d}, 1 \mathrm{H}, J=8.4) ; 6.81(\mathrm{~d}, 1 \mathrm{H}, J=2.6)$; $6.70(\mathrm{dd}, 1 \mathrm{H}, J=2.6,8.4) ; 6.31-6.30(\mathrm{~m}, 1 \mathrm{H}) ; 6.24-6.20(\mathrm{~m}, 1 \mathrm{H}) ; 3.79(\mathrm{~s}, 3 \mathrm{H}) ; 3.70(\mathrm{q}, 2 \mathrm{H}$, $J=13.8) ; 2.98(\mathrm{~d}, 1 \mathrm{H}, J=18.3) ; 2.85-2.81(\mathrm{~m}, 1 \mathrm{H}) ; 2.63(\mathrm{dd}, 1 \mathrm{H}, J=5.3,18.2) ; 2.55-2.50$ $(\mathrm{m}, 1 \mathrm{H}) ; 2.33(\mathrm{~d}, 1 \mathrm{H}, J=12.8) ; 2.17-2.08(\mathrm{~m}, 1 \mathrm{H}) ; 1.89(\mathrm{~d}, 1 \mathrm{H}, J=12.4) ; 1.83-1.73(\mathrm{~m}, 1 \mathrm{H})$; $1.63-1.60(\mathrm{~m}, 1 \mathrm{H}) ; 1.52-1.38(\mathrm{~m}, 1 \mathrm{H}) ; 1.35-1.29(\mathrm{~m}, 5 \mathrm{H}) ; 1.11$ (qd, $1 \mathrm{H}, J=3.6,12.4) .{ }^{13} \mathrm{C}$ NMR $\left(\mathrm{CDCl}_{3}, 100 \mathrm{MHz}\right): \delta 158.3,142.3,140.0,129.8,128.6,111.2,110.8,110.2,108.5,55.9,55.3$, $51.9,45.8,44.9,41.8,37.9,36.7,27.0,26.9,26.7,24.1,22.4$. HRMS (ESI): $m / z$ calculated for $\mathrm{C}_{22} \mathrm{H}_{28} \mathrm{NO}_{2}{ }^{+}[\mathrm{M}+\mathrm{H}]^{+}=338.2115$. Found $=338.2116$.

Cpd. 16h, $(9 \alpha, 13 \alpha, 14 \alpha)$-17-(2'-thiophenylmethyl)-3-methoxymorphinan, $\mathrm{C}_{22} \mathrm{H}_{27} \mathrm{NOS}$. ${ }^{1} \mathrm{H} \mathrm{NMR}\left(\mathrm{CDCl}_{3}, 400 \mathrm{MHz}\right): \delta 7.22(\mathrm{~d}, 1 \mathrm{H}, J=3.6) ; 7.04(\mathrm{~d}, 1 \mathrm{H}, J=8.4) ; 6.92(\mathrm{~d}, 1 \mathrm{H}, J=2.4)$; $6.81(\mathrm{~d}, 1 \mathrm{H}, J=2.4) ; 6.71(\mathrm{dd}, 1 \mathrm{H}, J=2.4,8.4) ; 3.94-3.78(\mathrm{~m}, 2 \mathrm{H}) ; 3.79(\mathrm{~s}, 3 \mathrm{H}) ; 3.01-2.91$ $(\mathrm{m}, 2 \mathrm{H}) ; 2.64(\mathrm{dd}, 1 \mathrm{H}, J=4.6,17.8) ; 2.55(\mathrm{~d}, 1 \mathrm{H}, J=9.2) ; 2.34(\mathrm{~d}, 1 \mathrm{H}, J=12.8) ; 2.13(\mathrm{t}, 1 \mathrm{H}$, $J=11.7) ; 1.87(\mathrm{~d}, 1 \mathrm{H}, J=11.6) ; 1.79-1.68(\mathrm{~m}, 1 \mathrm{H}) ; 1.62(\mathrm{~d}, 1 \mathrm{H}, J=9.2) ; 1.52(\mathrm{~d}, 1 \mathrm{H}, J=9.2)$; 1.41-1.24 (m, 6H); 1.09 (qd, $1 \mathrm{H} \mathrm{J}=3.4,12.5) .{ }^{13} \mathrm{C}$ NMR $\left(\mathrm{CDCl}_{3}, 100 \mathrm{MHz}\right): \delta 158.4,142.1$, 130.1, 128.6, 128.0, 126.5, 125.1, 124.8, 111.3, 110.8, 56.0, 55.3, 54.1, 45.6, 45.1, 42.1, 38.0, 36.7, $26.9,26.8,24.7,22.4$. HRMS (ESI): $m / z$ calculated for $\mathrm{C}_{22} \mathrm{H}_{28} \mathrm{NOS}^{+}[\mathrm{M}+\mathrm{H}]^{+}=354.1885$. Found $=354.1885$.

Cpd. 16i, $(9 \alpha, 13 \alpha, 14 \alpha)$-17-(2'-pyrrolylmethyl)-3-methoxy morphinan, $\mathrm{C}_{22} \mathrm{H}_{28} \mathrm{~N}_{2} \mathrm{O}$. ${ }^{1} \mathrm{H} \mathrm{NMR}\left(\mathrm{CDCl}_{3}, 400 \mathrm{MHz}\right): \delta 8.75(\mathrm{ls}, 1 \mathrm{H}) ; 7.04(\mathrm{~d}, 1 \mathrm{H}, J=8.4) ; 6.81(\mathrm{~d}, 1 \mathrm{H}, J=2.4) ; 6.75(\mathrm{q}$, $1 \mathrm{H}, J=1.4) ; 6.70(\mathrm{dd}, 1 \mathrm{H}, J=2.6,8.4) ; 6.12(\mathrm{q}, 1 \mathrm{H}, J=2.8) ; 6.03-6.00(\mathrm{~m}, 1 \mathrm{H}) ; 3.79(\mathrm{~s}, 3 \mathrm{H})$; $3.70(\mathrm{q}, 2 \mathrm{H}, J=13.8), 2.97(\mathrm{~d}, 1 \mathrm{H}, J=18.1) ; 2.83-2.80(\mathrm{~m}, 1 \mathrm{H}), 2.63(\mathrm{dd}, 1 \mathrm{H}, J=5.8,18.2)$; $2.46(\mathrm{dd}, 1 \mathrm{H}, J=3.2,12.0) ; 2.38-2.33(\mathrm{~m}, 1 \mathrm{H}) ; 2.13(\mathrm{td}, 1 \mathrm{H}, J=3.0,12.4) ; 1.85-1.79(\mathrm{~m}, 1 \mathrm{H})$; 1.72-1.62 (m, 2H); $1.55-1.49(\mathrm{~m}, 1 \mathrm{H}) ; 1.38-1.30(\mathrm{~m}, 5 \mathrm{H}) ; 1.15-1.09(\mathrm{~m}, 1 \mathrm{H}) .{ }^{13} \mathrm{C} \mathrm{NMR}\left(\mathrm{CDCl}_{3}\right.$, $100 \mathrm{MHz}): \delta 158.3,141.9,129.9,129.4,128.6,117.3,111.3,110.8,108.0,106.9,55.8,55.3,52.0$, 45.6, 45.3, 42.1, 38.0, 36.8, 26.9, 26.7, 24.5, 22.4. HRMS (ESI): $m / z$ calculated for $\mathrm{C}_{22} \mathrm{H}_{29} \mathrm{~N}_{2} \mathrm{O}^{+}$ $[\mathrm{M}+\mathrm{H}]^{+}=337.2274$. Found $=337.2275$.

Cpd. 16j, $(9 \alpha, 13 \alpha, 14 \alpha)$-17-(N-methyl-2'-pyrrolylmethyl)-3-methoxymorphinan, $\mathrm{C}_{23} \mathrm{H}_{30} \mathrm{~N}_{2} \mathrm{O} .{ }^{1} \mathrm{H}$ NMR $\left(\mathrm{CDCl}_{3}, 600 \mathrm{MHz}\right): \delta 7.06(\mathrm{~d}, 1 \mathrm{H}, J=8.3) ; 6.81(\mathrm{~d}, 1 \mathrm{H}, J=1.9) ; 6.72$ $(\mathrm{dd}, 1 \mathrm{H} J=1.9,8.3) ; 6.60(\mathrm{~s}, 1 \mathrm{H}) ; 6.02(\mathrm{~s}, 1 \mathrm{H}) ; 5.97(\mathrm{~s}, 1 \mathrm{H}) ; 3.78(\mathrm{~s}, 3 \mathrm{H}) ; 3.68-3.54(\mathrm{~m}, 5 \mathrm{H})$; $3.99(\mathrm{~d}, 1 \mathrm{H}, J=18.0) ; 2.79-2.76(\mathrm{~m}, 1 \mathrm{H}) ; 2.62-2.57(\mathrm{~m}, 1 \mathrm{H}) ; 2.46-2.43(\mathrm{~m}, 1 \mathrm{H}) ; 2.36-2.33(\mathrm{~m}$, $1 \mathrm{H}) ; 2.09-2.04(\mathrm{~m}, 1 \mathrm{H}) ; 1.76-1.73(\mathrm{~m}, 1 \mathrm{H}) ; 1.65-1.62(\mathrm{~m}, 2 \mathrm{H}) ; 1.52-1.50(\mathrm{~m}, 1 \mathrm{H}) ; 1.39-1.29(\mathrm{~m}$, 5H); 1.18-1.07 (m, 1H). ${ }^{13} \mathrm{C} \mathrm{NMR}\left(\mathrm{CDCl}_{3}, 150 \mathrm{MHz}\right): \delta 158.3,142.1,130.3,130.0,128.6,122.5$, $111.3,110.7,109.0,106.1,55.4,55.3,53.5,51.2,45.5,45.1,42.3,38.0,36.8,34.0,26.7,24.2,22.4$. HRMS (ESI): $m / z$ calculated for $\mathrm{C}_{23} \mathrm{H}_{31} \mathrm{~N}_{2} \mathrm{O}^{+}[\mathrm{M}+\mathrm{H}]^{+}=351.2431$. Found $=351.2431$.

Cpd. 16k, $(9 \alpha, 13 \alpha, 14 \alpha)$-17-(4"-methyl-5'-imidazolylmethyl)-3-methoxymorphinan, $\mathrm{C}_{22} \mathrm{H}_{29} \mathrm{~N}_{3} \mathrm{O} .{ }^{1} \mathrm{H}$ NMR $\left(\mathrm{CDCl}_{3}, 400 \mathrm{MHz}\right): \delta 7.44(\mathrm{~s}, 1 \mathrm{H}) ; 7.00(\mathrm{~d}, 1 \mathrm{H}, J=8.4) ; 6.74(\mathrm{~d}, 1 \mathrm{H}$, $J=2.4) ; 6.45(\mathrm{dd}, 1 \mathrm{H}, J=2.4,8.4) ; 3.72(\mathrm{~s}, 3 \mathrm{H}), 3.63(\mathrm{q}, 2 \mathrm{H}, J=13.6) ; 2.92(\mathrm{~d}, 1 \mathrm{H}, J=18.4)$; 2.82-2.78 (m, 1H); $2.63(\mathrm{dd}, 1 \mathrm{H}, J=5.2,18.0) ; 2.53-2.45(\mathrm{~m}, 1 \mathrm{H}) ; 2.30-2.26(\mathrm{~m}, 1 \mathrm{H}) ; 2.15(\mathrm{~s}$, $3 \mathrm{H}) ; 1.81-1.78(\mathrm{~m}, 1 \mathrm{H}) ; 1.71-1.66(\mathrm{~m}, 1 \mathrm{H}) ; 1.58-1.56(\mathrm{~m}, 1 \mathrm{H}) ; 1.47-1.44(\mathrm{~m}, 1 \mathrm{H}) ; 1.36(\mathrm{~s}, 3 \mathrm{H})$; 
$1.30-1.19(\mathrm{~m}, 4 \mathrm{H}) ; 1.17-0.98(\mathrm{~m}, 1 \mathrm{H})$. HRMS (ESI): $m / z$ calculated for $\mathrm{C}_{22} \mathrm{H}_{30} \mathrm{~N}_{3} \mathrm{O}^{+}[\mathrm{M}+$ $\mathrm{H}]^{+}=352.2383$. Found $=352.2378$.

Cpd. 161, $(9 \alpha, 13 \alpha, 14 \alpha)$-17-(2'-indolylmethyl)-3-methoxymorphinan, $\mathrm{C}_{26} \mathrm{H}_{30} \mathrm{~N}_{2} \mathrm{O}$. ${ }^{1} \mathrm{H} \mathrm{NMR}\left(\mathrm{CDCl}_{3}, 400 \mathrm{MHz}\right): \delta 8.79(\mathrm{ls}, 1 \mathrm{H}) ; 7.54(\mathrm{~d}, 1 \mathrm{H}, J=8.0) ; 7.36(\mathrm{~d}, 1 \mathrm{H}, J=8.0)$; $7.15(\mathrm{t}, 1 \mathrm{H}, J=7.2) ; 7.07(\mathrm{t}, 1 \mathrm{H}, J=6.8) ; 6.81(\mathrm{~d}, 1 \mathrm{H}, J=2.0) ; 6.72(\mathrm{dd}, 1 \mathrm{H}, J=2.4,8.4) ; 6.33$ $(\mathrm{s}, 1 \mathrm{H}) ; 3.88(\mathrm{q}, 2 \mathrm{H}, J=13.6) ; 3.81(\mathrm{~s}, 3 \mathrm{H}) ; 3.01(\mathrm{~d}, 1 \mathrm{H}, J=18.4) ; 2.87-2.43(\mathrm{~m}, 1 \mathrm{H}), 2.67(\mathrm{dd}$, $1 \mathrm{H}, J=5.6,18.0) ; 2.51(\mathrm{dd}, 1 \mathrm{H}, J=3.2,12.0) ; 2.33(\mathrm{~d}, 1 \mathrm{H}, J=12.8) ; 2.21(\mathrm{td}, 1 \mathrm{H}, J=3.2$, 12.0); $1.91-1.87(\mathrm{~m}, 1 \mathrm{H}) ; 1.74(\mathrm{td}, 1 \mathrm{H}, J=3.2,12.4) ; 1.65-1.62(\mathrm{~m}, 1 \mathrm{H}) ; 1.54-1.51(\mathrm{~m}, 1 \mathrm{H})$; 1.40-1.32 (m, 5H); $1.26(\mathrm{t}, 1 \mathrm{H}, J=7.2) ; 1.15-1.05(\mathrm{~m}, 1 \mathrm{H}) .{ }^{13} \mathrm{C} \mathrm{NMR}\left(\mathrm{CDCl}_{3}, 100 \mathrm{MHz}\right): \delta$ $158.3,136.1,128.6,128.5,121.5,120.1,119.6,111.2,110.8,56.0,55.2,52.3,45.7,41.8,37.8,36.6$, 26.7, 26.5, 24.6, 22.2. HRMS (ESI): $m / z$ calculated for $\mathrm{C}_{26} \mathrm{H}_{31} \mathrm{~N}_{2} \mathrm{O}^{+}[\mathrm{M}+\mathrm{H}]^{+}=387.2436$. Found $=387.2425$.

Cpd. 16m, $(9 \alpha, 13 \alpha, 14 \alpha)-17-\left(5^{\prime}-\left(2^{\prime}, 2^{\prime \prime}\right.\right.$-bithiophenyl)methyl)-3-methoxymorphinan, $\mathrm{C}_{26} \mathbf{H}_{29} \mathrm{NOS}_{2} .{ }^{1} \mathrm{H}$ NMR $\left(\mathrm{CDCl}_{3}, 400 \mathrm{MHz}\right): \delta 7.18(\mathrm{~d}, 1 \mathrm{H}, J=5.2) ; 7.14(\mathrm{~d}, 1 \mathrm{H}, J=3.2) ; 7.06$ $(\mathrm{d}, 1 \mathrm{H}, J=8.4) ; 7.01-6.98(\mathrm{~m}, 2 \mathrm{H}) ; 6.82(\mathrm{~d}, 1 \mathrm{H}, J=2.0) ; 6.72(\mathrm{dd}, 1 \mathrm{H}, J=2.4,8.4) ; 3.92-3.81$ (m, 2H); $3.82(\mathrm{~s}, 3 \mathrm{H}) ; 2.99-2.95(\mathrm{~m}, 2 \mathrm{H}) ; 2.71-2.66(\mathrm{~m}, 2 \mathrm{H}) ; 2.35(\mathrm{~d}, 1 \mathrm{H}, J=12.8) ; 2.07-2.03(\mathrm{~m}$, $1 \mathrm{H}) ; 1.92-1.89(\mathrm{~m}, 1 \mathrm{H}) ; 1.82-1.78(\mathrm{~m}, 1 \mathrm{H}) ; 1.64-1.62(\mathrm{~m}, 1 \mathrm{H}) ; 1.54(\mathrm{~d}, 1 \mathrm{H}, J=11.6) ; 1.41-1.24$ $(\mathrm{m}, 6 \mathrm{H}) ; 1.10(\mathrm{qd}, 1 \mathrm{H}, J=4.0,12.4)$. HRMS (ESI): $\mathrm{m} / z$ calculated for $\mathrm{C}_{26} \mathrm{H}_{30} \mathrm{NOS}_{2}{ }^{+}[\mathrm{M}+\mathrm{H}]^{+}$ $=436.1769$. Found $=436.1757$.

Cpd. 17, $(9 \alpha, 13 \alpha, 14 \alpha)$-17-cyclopropylcarbonyl-3-methoxymorphinan, $\mathrm{C}_{21} \mathrm{H}_{27} \mathrm{NO}_{2}$. To solution of nor-DXM $15(36 \mathrm{mg}, 0.140 \mathrm{mmol})$ in $\mathrm{CH}_{2} \mathrm{Cl}_{2}(500 \mu \mathrm{L})$ at $0^{\circ} \mathrm{C}$ was added cyclopropanecarbonyl chloride $(16 \mathrm{mg}, 0.153 \mathrm{mmol})$ then $\mathrm{NEt}_{3}(20 \mu \mathrm{L})$. The reaction mixture was allowed to warm to $\mathrm{r}$. $\mathrm{t}$. and further stirred for $1.5 \mathrm{~h}$. The mixture was treated with $\mathrm{NaHCO}_{3} / \mathrm{Na}_{2} \mathrm{CO}_{3}$ buffer $(\mathrm{pH} 9.5,500 \mu \mathrm{L}$ ), the organic layer was separated, dried over $\mathrm{MgSO}_{4}$, filtered and evaporated under reduced pressure. The crude product was purified by column chromatography on silica gel using EtOAc/cyclohexane (0:100 to 20:80 $\mathrm{v} / \mathrm{v})$ as eluent to afford $17(34 \mathrm{mg}, 75 \%) .{ }^{1} \mathrm{H}$ NMR $\left(\mathrm{CDCl}_{3}, 400 \mathrm{MHz}\right): \delta 7.02(\mathrm{~d}, 1 \mathrm{H}, J=8.4) ; 6.84(\mathrm{~d}$, $1 \mathrm{H}, J=2.6) ; 6.72(\mathrm{dd}, 1 \mathrm{H}, J=2.6,8.4) ; 4.76-4.65(\mathrm{~m}, 1 \mathrm{H}) ; 4.09-4.03(\mathrm{~m}, 1 \mathrm{H},) ; 3.79(\mathrm{~s}, 3 \mathrm{H}) ; 3.13$ $(\mathrm{dd}, 1 \mathrm{H}, J=6.0,18.0) ; 2.66(\mathrm{~d}, 1 \mathrm{H}, J=17.6) ; 2.40-2.36(\mathrm{~m}, 1 \mathrm{H}) ; 1.74-1.24(\mathrm{~m}, 11 \mathrm{H}) ; 1.14-1.04$

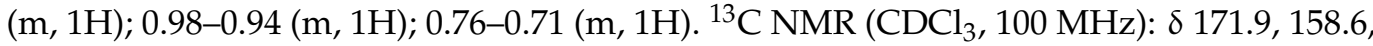
140.6, 129.2, 128.5, 111.5, 111.3, 55.4, 44.2, 42.1, 38.0, 36.6, 31.6, 26.7, 22.2, 11.6, 7.4, 7.2. HRMS (ESI): $m / z$ calculated for $\mathrm{C}_{21} \mathrm{H}_{27} \mathrm{NO}_{2} \mathrm{Na}^{+}[\mathrm{M}+\mathrm{Na}]^{+}=348.1934$. Found $=348.1935$.

Cpd. $18,(9 \alpha, 13 \alpha, 14 \alpha)-17,17-d i m e t h y l-3-m e t h o x y$ morphinan iodide, $\mathrm{C}_{19} \mathrm{H}_{28} \mathrm{NOI}$. To a solution of DXM 3 free base $(30 \mathrm{mg} 0.11 \mathrm{mmol})$ in $\mathrm{CH}_{2} \mathrm{Cl}_{2}(300 \mu \mathrm{L}), \mathrm{CH}_{3} \mathrm{I}(300 \mu \mathrm{L}, 4.82$ $\mathrm{mmol}$ ) was added, and the mixture was stirred for $2 \mathrm{~h}$ at $\mathrm{r}$. t. The obtained white precipitate was filtered and washed with EtOAc $(4 \times 300 \mu \mathrm{L})$ to produce 18 as a white powder $(26 \mathrm{mg}$, $0.091 \mathrm{mmol}, 82 \%$ yield $).{ }^{1} \mathrm{H}$ NMR $\left(\mathrm{CDCl}_{3}, 400 \mathrm{MHz}\right): \delta 7.12(\mathrm{~d}, 1 \mathrm{H}, J=9.2) ; 6.80-6.77(\mathrm{~m}$, $2 \mathrm{H}) ; 4.10-4.07(\mathrm{~m}, 1 \mathrm{H}) ; 3.58(\mathrm{~s}, 3 \mathrm{H}) ; 3.69(\mathrm{~s}, 3 \mathrm{H}) ; 3.61-3.55(\mathrm{~m}, 1 \mathrm{H}) ; 3.52(\mathrm{~s}, 3 \mathrm{H}) ; 3.38-3.32(\mathrm{~m}$, $1 \mathrm{H}) ; 2.87-2.85(\mathrm{~m}, 1 \mathrm{H}) ; 2.43-2.36(\mathrm{~m}, 2 \mathrm{H}) ; 2.14-2.12(\mathrm{~m}, 1 \mathrm{H}) ; 1.74-1.44(\mathrm{~m}, 6 \mathrm{H}) ; 1.33-1.22$ $(\mathrm{m}, 1 \mathrm{H}) ; 1.12(\mathrm{qd}, 1 \mathrm{H}, J=3.6,12.4) .{ }^{13} \mathrm{C}$ NMR $\left(\mathrm{CDCl}_{3}, 100 \mathrm{MHz}\right): \delta 159.7,138.6,129.5,124.1$, $112.6,111.4,69.6,57.7,55.5,54.5,51.2,38.7,36.8,36.5,35.5,26.7,26.4,25.9,21.8$. HRMS (ESI): $m / z$ calculated for $\mathrm{C}_{19} \mathrm{H}_{28} \mathrm{NO}^{+}\left[\mathrm{M}^{+}\right]=286.2171$. Found $=286.2166$.

Drug preparation and storage. Tazopsine 1, DXM 3, and derivatives (cpds. 6-18) were prepared in DMSO at $10 \mathrm{mM}$, aliquoted, then stored at $-20^{\circ} \mathrm{C}$. PQ and CQ biphosphates were prepared at $110 \mathrm{mM}$ and $132 \mu \mathrm{M}$, respectively, in sterile water, aliquoted, then stored at $-20^{\circ} \mathrm{C}$. A stock aliquot was thawed and used for daily medium changes of the parasite cultures whenever necessary.

\subsection{Parasite Maintenance and Inhibition Assays (By Order of Appearance in the Manuscript) \\ 2.3.1. P. yoelii Growth Inhibition Assays In Vitro}

Parasite culture. Primary mouse hepatocytes were isolated as previously described [21] and seeded at $10^{5}$ cells per well in eight-well Lab-Tek plastic chamber slides (VWR, Fontenay-sous-Bois, France) previously coated with rat tail collagen I (BD Biosciences, Le Pont de Claix, France). Mouse hepatocytes were cultured at $37^{\circ} \mathrm{C}$ in 
$5 \% \mathrm{CO}_{2}$ in complete William's E medium (10\% fetal calf serum, $1 \%$ L-glutamine, $1 \%$ sodium pyruvate, $1 \%$ insulin-transferrin-selenium, $1 \%$ non-essential amino acids, and $1 \%$ penicillin-streptomycin, all obtained from Invitrogen, Cergy-Pontoise, France). After $24 \mathrm{~h}, 10^{5}$ P. yoelii (265 BY strain) sporozoites, isolated from infected Anopheles stephensi, were added per well. Compounds to be tested were solubilized in DMSO, further diluted in complete William's E medium (final DMSO concentrations $<0.3 \%$ ), and added to hepatocyte cultures at the time of sporozoite inoculation. Each cpd. concentration was tested in triplicate. Infected cultures were maintained for $1 \mathrm{~h}$ at $\mathrm{r}$. t. to allow parasites to settle down onto the hepatocytes, and were then transferred to the incubator at $37{ }^{\circ} \mathrm{C}$ in an atmosphere containing $5 \% \mathrm{CO}_{2}$. After $3 \mathrm{~h}$, cultures were washed and further incubated in the presence of each test cpd. Culture medium containing the appropriate cpd. concentration was renewed $24 \mathrm{~h}$ later, and cultures were fixed with cold methanol $48 \mathrm{~h}$ post-infection.

IC $_{50}$ measurement. After fixation of hepatocyte cultures with cold methanol, parasites were stained with an anti-P. falciparum heat shock protein 70 mouse polyclonal serum (crossreactive with $P$. yoelii) and revealed with FITC-conjugated goat anti-mouse IgG secondary antibody (Sigma-Aldrich). Parasites were counted under a fluorescence microscope with a $25 \times$ light objective. $\mathrm{IC}_{50}$ values, e.g., compound concentrations at which a $50 \%$ reduction in parasite number was observed compared to DMSO control, were calculated by linear regression using Excel 2016 software and derived from three independent experiments.

\subsubsection{P. yoelii Growth Inhibition Assays In Vivo}

These experiments were performed as described in Bosson Vanga et al. [22]. Briefly, 6to 8-week-old BALB/c female mice were used (Janvier CERJ, Le Genest-Saint-Isle, France) and housed at CEF (UMS28, La Pitié-Salpêtrière). All animal work was conducted in strict accordance with the European Parliament and Council Directive 2010/63/EU on the protection of animals used for scientific purposes. Protocols were approved by the Charles Darwin Ethics Committee for animal experimentation CEEA-005 (approval \#01736.02). Five mice were used per treatment group. Drugs were administrated on days $-1,0,+1$, and mice were infected on day 0 by intravenous injection of 10,000 P. yoelii-Luc sporozoites. In vivo imaging was performed $44 \mathrm{~h}$ post-infection to assess liver stage development with an IVIS Spectrum (Caliper Life Science, Hanover, MD, USA). Mice were injected intraperitoneally with D-luciferin $(100 \mathrm{mg} / \mathrm{kg})$, anesthetized with isoflurane, and imaged for bioluminescence 10 min post-luciferin injection. Data acquisition was conducted using Living Image software 3.0 (Caliper Life Sciences, Hanover, MD, USA). Data analysis and statistical analysis using a one-way ANOVA test for multiple comparisons were performed with GraphPad Prism 8 statistical software (GraphPad. Software, San Diego, CA, USA).

\subsubsection{P. falciparum and P. berghei Liver Stages Growth Inhibition Assays In Vitro}

Parasite culture. Plasmodium liver stages were cultured as described elsewhere (Baron et al. manuscript in preparation). Briefly, cryopreserved primary human hepatocytes were purchased from Lonza Bioscience and Biopredic International (Saint-Grégoire, France). Cells were thawed and seeded into 384-well plates (Greiner Bio-One, Germany) pre-coated with rat tail collagen I (BD Bioscience, Le Pont de Claix, France). Human hepatocytes were maintained at $37^{\circ} \mathrm{C}$ in $5 \% \mathrm{CO}_{2}$ in William's E medium (Gibco) supplemented with $10 \%$ fetal clone III serum (HyClone, Dutscher, Bernolsheim, France), $100 \mu \mathrm{g} / \mathrm{mL}$ penicillin and $100 \mu \mathrm{g} / \mathrm{mL}$ streptomycin (ThermoFisher Scientific, Courtaboeuf, France), $5 \times 10^{-3}$ $\mathrm{g} / \mathrm{L}$ human insulin (Sigma-Aldrich, Saint Quentin Fallavier, France), and $5 \times 10^{-5} \mathrm{M}$ hydrocortisone (Upjohn Laboratories SERB, France). The next day, cells were overlaid with matrigel (Corning) and the medium was then renewed every two days. Four days later, sporozoites were isolated by aseptic hand dissection of salivary glands of $P$. bergheiGFP [23] or P. falciparum-infected mosquitoes ( $P$. falciparum NF54 strain, obtained from the Department of Medical Microbiology, University Medical Centre, St Radboud, Nijmegen, The Netherlands). Matrigel was then removed from the hepatocyte culture, and 5000 or 
30,000 sporozoites of $P$. berghei-GFP or P. falciparum, respectively, were inoculated into cells before centrifugation at $560 \mathrm{xg}$ for $10 \mathrm{~min}$ at $\mathrm{r}$. $\mathrm{t}$. and subsequent incubation at $37^{\circ} \mathrm{C}$ and $5 \% \mathrm{CO}_{2}$. Drugs were tested in quadruplicate, starting from time of sporozoite addition. After $3 \mathrm{~h}$, infected cultures were covered with matrigel prior to addition of fresh cell culture medium containing the appropriate drug dilutions. Media, containing drugs or not, were renewed on a daily basis until cell fixation, which occurred at $48 \mathrm{~h}$ and 6 days post-infection for P. berghei and P. falciparum sporozoites, respectively.

Immunostaining of liver stages. Infected cultures were fixed using $4 \%$ paraformaldehyde (PFA) for $15 \mathrm{~min}$ at r. t., and liver stage parasites were immune-labeled with polyclonal anti-PfHSP70 murine serum and revealed with Alexa-Fluor 488-conjugated goat anti-mouse immunoglobulin (Invitrogen). DAPI was used to visualize nuclei.

Parasite enumeration and toxicity assessment using high-content imaging. A CellInsight High-Content Screening Platform and Studio HCS software (Thermo Fisher Scientific, Waltham, MA, USA) were used to determine parasite number and size in fixed cultures. Reduction in parasite size was calculated based on the average object area, as described previously [24]. Compound cytotoxicity was determined by counting DAPI-positive host cell nuclei.

IC $_{50}$ measurement. $\mathrm{IC}_{50}$ values were determined by non-linear regression with GraphPad Prism 8 software. The logarithm of concentration was expressed as a function of the parasites number normalized to the drug free controls. The tests on 384-well plates were conducted in quadruplicates.

Statistical Analysis. GraphPad Prism 8 statistical software (GraphPad. Software, San Diego, CA, USA) and Excel 2016 software (Microsoft Office) were used in this study for the data analysis. All graph values represent means and error bars represent standard deviations (s. d.).

\subsubsection{P. falciparum Asexual Blood Stages}

Parasite culture. Chloroquine-sensitive (3D7) P. falciparum strain was obtained from the Malaria Research and Reference Reagent Resource Center (MR4). Parasites were maintained in human erythrocytes $\left(\mathrm{O}^{+}\right.$, provided by Etablissement français du sang, EFS, Rungis, France), at 5\% hematocrit, suspended in complete culture medium RPMI 1640 supplemented with $25 \mathrm{mM}$ HEPES, $20 \mathrm{mM}$ D-glucose, $25 \mathrm{mM}$ sodium bicarbonate, $0.4 \mathrm{mM}$ hypoxanthine, $5 \mathrm{mM}$ L-glutamine, and 10\% AB human serum. Parasite cultures were maintained at $37{ }^{\circ} \mathrm{C}$ in a gaseous environment composed of $5 \% \mathrm{CO}_{2}, 10 \% \mathrm{O}_{2}$, and $85 \% \mathrm{~N}_{2}$. The culture medium was changed daily. Parasitemia was controlled using light microscopy (Axioskop microscope, ZEISS, Oberkochen, Germany) under oil immersion, after fixing thin blood smears with methanol and staining with Diff-Quik ${ }^{\mathrm{TM}}$ stain set (RAL Diagnostics, Martillac, France).

IC $_{50}$ measurement. A 50\% inhibitory concentrations ( $\left.\mathrm{IC}_{50}\right)$ determination test was carried out using isotopic ${ }^{3} \mathrm{H}$-hypoxanthine incorporation assays, as previously described [25], with minor modifications. Briefly, P. falciparum cultures at ring stage were highly synchronized by two consecutive treatments with 5\% sorbitol (Sigma-Aldrich) in PBS (v/v) at $40 \mathrm{~h}$ intervals and diluted down to $0.3-0.5 \%$ parasitemia and $2 \%$ hematocrit. Parasites were dispensed into 96-well plates containing 14 serially diluted drug concentrations ranging from 0 to $240 \mu \mathrm{M}$, and incubated as described above in the presence of $5 \%{ }^{3} \mathrm{H}$-hypoxanthine (Perkin Elmer, Waltham, Massachusetts, USA) for $42 \mathrm{~h}$. Next, ${ }^{3} \mathrm{H}$-hypoxanthine uptake was evaluated by scintillation counting (Top Count NXT, Perkin Elmer, Waltham, Massachusetts, USA) and results were expressed as the inhibitory concentrations $\left(\mathrm{IC}_{50}\right)$ defined as drug concentrations at which $50 \%$ of ${ }^{3} \mathrm{H}$-hypoxanthine incorporation was inhibited compared with drug-free controls. IC $_{50}$ values were established by non-linear regression with ICEstimator software (http:/ / www.antimalarial-icestimator.net/ 28 December 2021) [26,27]. The tests on 96-well plates were conducted in triplicate. 


\subsubsection{P. falciparum Sexual Blood Stages}

Parasite culture and gametocyte production. The $P$. falciparum transgenic line NF54cg6-Pfs16-CBG99 has been described elsewhere [28,29]. Parasite cultures were grown in human erythrocytes at 5\% hematocrit and RPMI 1640 media supplemented with hypoxanthine and $10 \%$ heat-inactivated human serum. Synchronization of asexual stages was achieved by magnetic isolation of schizonts from the culture, followed by depletion of schizonts several hours later, using a MACS depletion column (Miltenyi Biotec) in conjunction with a magnetic separator. To obtain synchronous gametocytes, cultures at 10-15\% ring stages were treated with $50 \mathrm{mM}$-acetylglucosamine (NAG) for 5 days to eliminate asexual parasites.

IC $_{50}$ measurement. To calculate the $\mathrm{IC}_{50}$ for DXM 3 and $N-2^{\prime}$-pyrrolylmethyl-norDXM 16i on early and mature gametocytes, $2 \times 10^{5}$ MACS-purified early GIE (day 2 post-NAG treatment) and mature GIE (day 7 post-NAG treatment) from the NF54-cg6Pfs16-CBG99 line were incubated with serial dilutions of inhibitors, or 2\% DMSO, for $72 \mathrm{~h}$. After $72 \mathrm{~h}$, GIE were washed and cell viability was evaluated by adding a non-lysing formulation of $0.5 \mathrm{mM}$ D-luciferin substrate [25], and by measuring luciferase activity for $1 \mathrm{~s}$ on an Infinite 200 PRO plate reader $\left(\operatorname{Tecan}^{\circledR}\right)$. The tests on 96 -well plates were performed in triplicate.

\section{Results}

\subsection{Extended SAR in the Tazopsine Series}

We present here pharmacomodulation efforts towards novel derivatives of tazopsine 1. Altogether, these SARs drove the validation of DXM 3 as a general and simplified mimic of all natural ent-morphinan alkaloids, and subsequently guided its chemical diversification into optimized antiplasmodial derivatives. Tazopsine $\mathbf{1}$ was treated with excess diazomethane to produce the 4-methyl phenol ether 6 with a 39\% yield. The native alkaloid was, in parallel, submitted to reductive amination with various aldehydes in the presence of sodium cyanoborohydride, to deliver tertiary amines $7 \mathbf{a}-\mathbf{g}$ with $45-82 \%$ yields. To assess the influence of a basic nitrogen on the antiplasmodial activity, $N$-acetyl-tazopsine 8 was produced in a $45 \%$ yield by treating tazopsine 1 with acetic anhydride (Figure 2). 

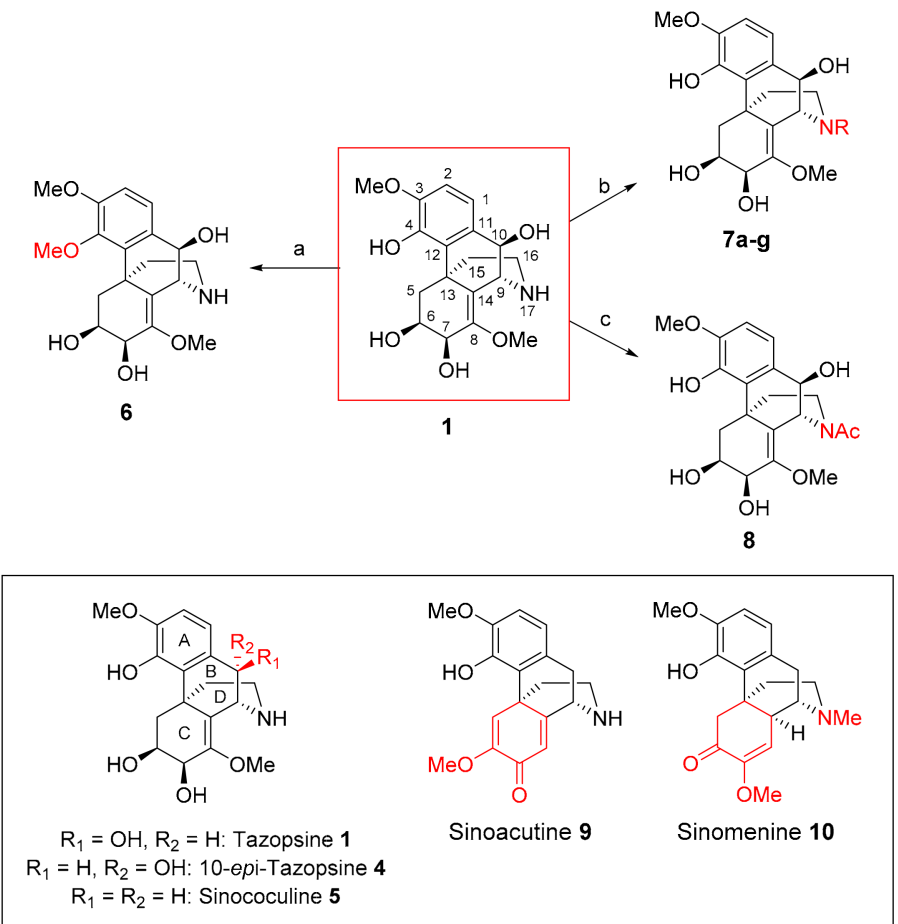

Figure 2. Semisynthetic access to tazopsine derivatives 6-8 and structure of natural ent-morphinan alkaloids for SAR generation (morphine numbering). (a) $\mathrm{CH}_{2} \mathrm{~N}_{2}, \mathrm{MeOH}, 0{ }^{\circ} \mathrm{C}, 12 \mathrm{~h}(39 \%)$; (b) aldehyde (1.1 eq), $\mathrm{MeOH}$, r. t., 10 min then $\mathrm{NaBH}_{3} \mathrm{CN}$ (1 eq), r. t. (45-82\%); (c) $\mathrm{Ac}_{2} \mathrm{O}$ (1 eq), $\mathrm{MeOH}$, r. t., $1 \mathrm{~h}(45 \%)$. The box indicates the natural alkaloids.

Primary mouse hepatocytes (PMH) infected by the murine parasite $P$. yoelii were used to assess the bioactivity of the generated tazopsine derivatives (Table 2), allowing a direct comparison with previously generated SARs in the series [14,15]. Benzylic 10-substitution (ring B) SAR: The comparison of tazopsine 1, 10-epi-tazopsine 4, and sinococuline 5 to assess the effect of benzylic 10-substitution, showed that the 10-(R)-hydroxy pattern of tazopsine 1 was optimal, its 10-epimer 4 being 5 -fold less active. However, an unsubstituted benzylic position proved to be only slightly less beneficial than when 10-(R) was hydroxylated, as showed by the comparable bioactivities of sinococuline 5 and tazopsine 1. Aromatic 4-O-substitution (ring A) SAR: The alkylation of the tazopsine 1 free phenol abolished the antiplasmodial activity, with 4-O-methyl-tazospine 6 showing non-significant inhibitory effects against the parasite even at $100 \mu \mathrm{M}$. 17-N-substitution (ring D) SAR: The tertiary amine derivatives of tazopsine 1 showed reduced activity with the increase in substituent size, $N$-methyl-tazopsine 7 a already being 2 -fold less active than the parent alkaloid, whereas the $\mathrm{N}-3^{\prime}, 4^{\prime}$-methylenedioxybenzyl congener exhibited abolished activity. However, this trend was mitigated by the beneficial $N$-4'-halo-benzyl substituents in analogues $7 \mathbf{f}$ and $7 \mathrm{~g}$, these exhibiting similar levels of inhibition to $\mathrm{N}$-methyl-tazopsine $7 \mathrm{a}$. This suggests that specific substituents can favorably impact the antiplasmodial activity of $\mathrm{N}$-modified entmorphinans despite relative bulkiness, as previously observed in $\mathrm{N}$-cyclopentyltazopsine 2 $\left(\mathrm{IC}_{50}=3.5 \pm 0.1 \mu \mathrm{M}\right)[14]$. On the other hand, $N$-acetyl-tazopsine 8 was completely devoid of activity, suggesting that the presence of a nitrogen atom, either basic (i.e., protonated at physiological $\mathrm{pH}$ values) or capable of engaging donating hydrogen bonds, was important for the antiplasmodial properties. Cyclohexenediol/cyclohexadienone/cyclohexenone (ring C) SAR: The comparison of tazopsine $\mathbf{1}$ with sinoacutine $\mathbf{9}$ and sinomenine $\mathbf{1 0}$ revealed that the southern portion of these alkaloids exerted a profound influence on their bioactivity. Indeed, only the 6,7-dihydroxy-8,14-methylenol moiety of tazopsine $\mathbf{1}$ correlated with strong antiplasmodial effects, while the distinctive methoxy-enone/dienone systems present in $\mathbf{9}$ and $\mathbf{1 0}$ led to abolished activity (Figure 2). In conclusion, regarding these antiplasmodial SARs in the ent-morphinan series, it appeared that the benzylic substitution 
at C-10 in ring B had to be either (R)-hydroxyl (as in tazopsine 1) or non-existent (as in sinococuline 5 and DXM 3). The ring $\mathrm{A}$ in tazopsine 1 seems to be a sensitive component to modify, considering the loss of activity exhibited by 4-O-methyltazopsine 6 . However, this punctual variation precludes a definitive conclusion. $N$-alkylation in the tazopsine series consistently appeared as a relevant pharmacomodulation at ring $D$, with frequent conservation of antiplasmodial activity. Lastly, the SAR regarding the southern ring C of ent-morphinans remains inconclusive, except for the restricted benefit of that present in tazopsine 1, a fact corroborated by the previous description of bioactivity loss in the tazopsine-6,7-acetonide [14].

Table 2. In vitro $\mathrm{IC}_{50}$ values of tazopsine 1, derivatives 6-8 and natural ent-morphinan alkaloids in $P$. yoelii liver stages. $\mathrm{IC}_{50}$ values are the mean of four technical replicates. PMH: primary mouse hepatocytes; NA: not applicable; PQ: primaquine.

\begin{tabular}{|c|c|c|}
\hline Cpd. & Substitutions & $\begin{array}{c}\mathrm{IC}_{50} \\
\text { (Py265BY-PMH, } \mu \mathrm{M})\end{array}$ \\
\hline Tazopsine 1 & $\mathrm{R}_{1}=\mathrm{OH}, \mathrm{R}_{2}=\mathrm{H}$ & $3.1 \pm 0.2$ \\
\hline 10-epi-tazopsine 4 & $\mathrm{R}_{1}=\mathrm{H}, \mathrm{R}_{2}=\mathrm{OH}$ & $16.1 \pm 1.9$ \\
\hline Sinococuline 5 & $\mathrm{R}_{1}=\mathrm{R}_{2}=\mathrm{H}$ & $4.5 \pm 0.4$ \\
\hline 4-O-Me-tazopsine 6 & NA & $>100$ \\
\hline$N$-methyl-tazopsine $\mathbf{7 a}$ & $\mathrm{R}=\mathrm{Me}$ & $5.8 \pm 0.4$ \\
\hline$N$-n-propyl-tazopsine $\mathbf{7 b}$ & $\mathrm{R}=n$-Pro & $12.6 \pm 1.7$ \\
\hline$N-4^{\prime}$-hydroxybenzyl-tazopsine $7 \mathrm{c}$ & $\mathrm{R}=4-\mathrm{OH}-\mathrm{Bn}$ & $14.2 \pm 2.2$ \\
\hline N-4'-methoxybenzyl-tazopsine $\mathbf{7 d}$ & $\mathrm{R}=4-\mathrm{OMe}-\mathrm{Bn}$ & $24.2 \pm 0.7$ \\
\hline
\end{tabular}

Table 2. Cont.

\begin{tabular}{|c|c|c|}
\hline Cpd. & Substitutions & $\begin{array}{c}\mathrm{IC}_{50} \\
\text { (Py265BY-PMH, } \mu \mathrm{M})\end{array}$ \\
\hline N-3', $4^{\prime}$-methylenedioxybenzyl-tazopsine $7 \mathrm{e}$ & $\mathrm{R}=3,4$-methylenedioxy-Bn & $>100$ \\
\hline$N-4^{\prime}$-chlorobenzyl-tazopsine $7 f$ & $\mathrm{R}=4-\mathrm{Cl}-\mathrm{Bn}$ & $5.8 \pm 1.1$ \\
\hline $\mathrm{N}-4^{\prime}$-bromobenzyl-tazopsine $\mathbf{7 g}$ & $\mathrm{R}=4-\mathrm{Br}-\mathrm{Bn}$ & $4.2 \pm 0.3$ \\
\hline $\mathrm{N}$-acetyl-tazopsine 8 & $\mathrm{R}=\mathrm{Ac}$ & $>100$ \\
\hline Sinoacutine 9 & NA & $>100$ \\
\hline Sinomenine 10 & NA & $>100$ \\
\hline PQ & NA & $0.62 \pm 0.03$ \\
\hline
\end{tabular}

\subsection{DXM Repurposing against Malaria}

DXM 3 is a well-known antitussive drug and pain reliever, also used as a dissociative anesthetic and hallucinogen in recreational use [30,31]. Its pharmacology in the central nervous system is well established. Despite being considered a synthetic opiate, DXM 3 does not act at the level of the opioid receptors, binding instead with high affinity to sigma receptors as an agonist, and to a lesser extent to the phencyclidine channel of $N$ methyl-D-aspartate (NMDA) receptors as an antagonist [31]. The relationship between the neuropharmacology and the antitussive effects of DXM 3 is poorly understood [30]. The similarity of DXM 3 with tazopsine 1 regarding their ent-morphinan backbone prompted us to evaluate its antiplasmodial properties both in vitro and in vivo, having in mind its possible direct repurposing against malaria. In a preliminary screening against primary human hepatocytes (PHH) infected by P. falciparum in vitro, DXM 3 exhibited an activity that was only 2 -fold less than that of tazopsine $\mathbf{1}$ (Table 3 ). It is noteworthy that the $\mathrm{IC}_{50}$ value of tazopsine 1 was double the previously described value of ca. $4 \mu \mathrm{M}$ in this same biological model [14]. This observation can be explained by the shift in the PHH used for $P$. falciparum culture from clinical samples in the previous study to standardized, commercially available cryopreserved PHH in the present work. The whole assay was validated by the expected submicromolar activity of the reference drug primaquine (PQ) [14]. 
Table 3. In vitro $\mathrm{IC}_{50}$ values of tazopsine $\mathbf{1}$ and DXM 3 in $P$. falciparum liver stages. $\mathrm{IC}_{50}$ values are the mean of four technical replicates. $\mathrm{PHH}$, primary human hepatocytes; $\mathrm{PQ}$, primaquine.

\begin{tabular}{cc}
\hline Cpds. & IC $\left._{\mathbf{5 0}} \mathbf{( P H H}, \boldsymbol{\mu M}\right)$ \\
\hline Tazopsine 1 & $7.88 \pm 3.05$ \\
\hline DXM 3 & $15.59 \pm 1.19$ \\
\hline PQ & $0.75 \pm 0.15$ \\
\hline
\end{tabular}

Following the exciting discovery of the antiplasmodial activity of DXM 3 against P. falciparum liver stages in vitro, we tested its prophylactic potential in a P. yoelii-infected mouse model of malaria. We used the transgenic parasite line P. yoelii-Luc infecting BALB/c mice, to follow parasitemia in situ based on the spontaneously emitted bioluminescence after injection of D-luciferin [32]. Following a preliminary study, we found that DXM 3 induced convulsive episodes and death in mice at doses higher than $60 \mathrm{mg} / \mathrm{kg}$ administered daily (data not shown). Therefore, we decided to use a subtoxic regimen of $40 \mathrm{mg} / \mathrm{kg}$ DXM 3 administered daily, starting $24 \mathrm{~h}$ before infection and further maintained for a $48 \mathrm{~h}$ period, corresponding to the duration of $P$. yoelii liver phase. In addition, we chose to associate DXM 3 with quinidine (QND), a known inhibitor of CYP2D6-mediated O-demethylation of DXM 3 into its main hepatic metabolite dextrorphan 12 (DX, syn. 3hydroxy-17-methylmorphinan) [31], in order to increase the plasmatic half-life of DXM $3[33,34]$ and possibly increase its antimalarial effect in vivo. DXM 3 at $40 \mathrm{mg} / \mathrm{kg}$ and QND at $20 \mathrm{mg} / \mathrm{kg}$ were deprived of prophylactic activity in this in vivo model, with parasitemia levels not significatively different between these groups and the vehicle (Figure 3). Although the combination of DXM 3 and QND at a daily regimen of $40 \mathrm{mg} / \mathrm{kg}$ and $20 \mathrm{mg} / \mathrm{kg}$, respectively, exerted significative prophylactic inhibition of $P$. yoelii-Luc growth, we found that this synergistic association was far from eliciting complete parasite clearance (vehicle vs. DXM40 + QND20: $p=0.5171$ ), as achieved by the reference drug primaquine biphosphate at a daily regimen of $5 \mathrm{mg} / \mathrm{kg}$ (vehicle vs. PQ: $p=0.0124$ ) (Figure 3).

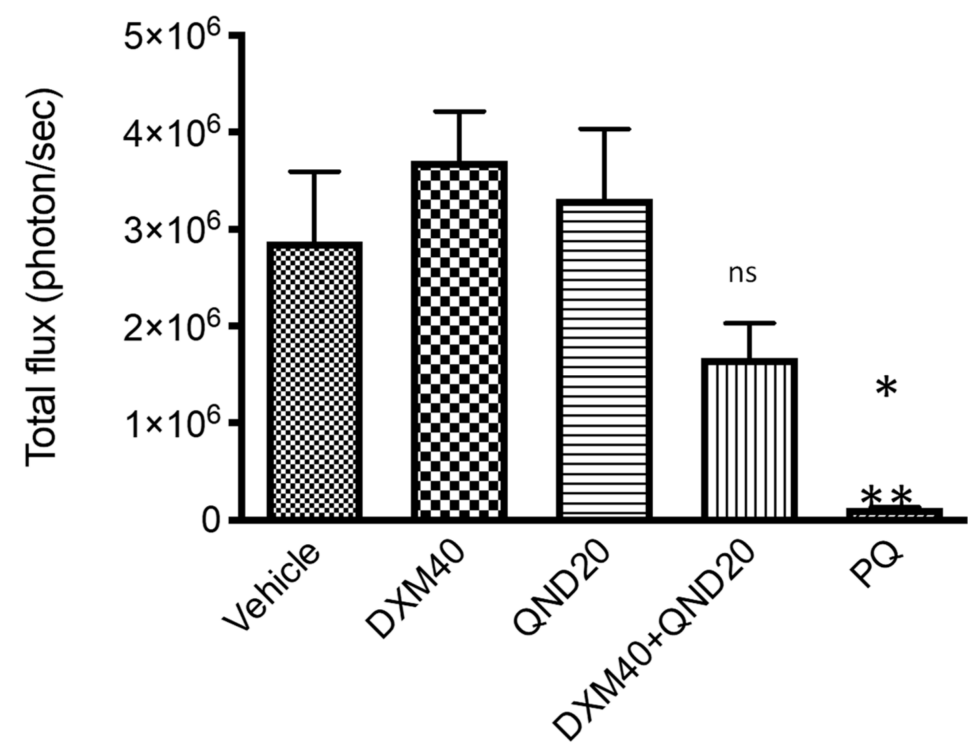

Figure 3. In vivo prophylactic activity of DXM 3 + / - QND compared to PQ in a mouse model of $P$. yoelii-Luc infection. Each group of mice consisted of five individuals. Results are shown as mean +/ - SEM: Vehicle vs. DXM40 + QND20, $p=0.5171$ (ns, not significative); Vehicle vs. PQ, $p=0.0124$ $\left.{ }^{*}\right)$; DXM40 vs. PQ and QND20 vs. PQ, $p=0.003\left({ }^{* *}\right)$. ANOVA one-way test for multiple comparisons. Numeric values indicate doses in $\mathrm{mg} / \mathrm{kg}$.

The extent of DXM 3 metabolization in vivo into DX 12 (theoretically inhibited by QND) and to a lesser degree into nor-DXM 15 (3-methoxymorphinan) by CYP3A4-mediated 
$\mathrm{N}$-demethylation $[31,35]$ remains uncharacterized in our study. If effective and yielding inactive metabolites, its occurrence could explain the relatively poor prophylactic activity of DXM 3 in this mouse model of malaria. However, this outcome proved to be invalid, as both DX 12 and nor-DXM 15 were later synthesized (Figure 4) and found to have similar levels of inhibitory potency in vitro to DXM 3 against $P$. berghei (Figures 5 and 6). Despite the unlikeliness of its repurposing as a prophylactic drug against malaria, DXM 3 represents a readily accessible and flexible synthetic platform upon which to explore the antimalarial potential of the ent-morphinan series, and circumvent the inherent limitations of the tazopsines.

\subsection{DXM Pharmacomodulation towards Improved Antiplasmodial Derivatives}

DXM 3, possessing the same backbone as the natural hit tazopsine 1, constituted the synthetic starting point of our study. This compound combines two advantages for a SAR study: (i) straightforward and cheap access from various commercial suppliers in gram quantities, and (ii) facile functionalization on the C-2 and/or $\mathrm{O}$ - and/or $\mathrm{N}$-positions (Figure 4). To explore yet unraveled SARs on the aryl ring, DXM 3 was firstly $o$-iodinated using $N$-iodosuccinimide (NIS) to produce 2-I-DXM 11 with a $91 \%$ yield. DX 12 was obtained as described by Jakobsson et al. [17] by the O-demethylation of DXM 3 with $48 \%$ aqueous $\mathrm{HBr}$, then $o$-iodinated with NIS under the previous conditions to produce 2-I-DX 13 with a $87 \%$ yield (Figure 4). Alkylation of the secondary amine position of tazopsine 1 constituting a relevant modification retaining the antiplasmodial activity of derivatives (Table 2) and possibly improving their antimalarial profile [14], $\mathrm{N}$-modification of norDXM 15 was further explored. Towards this aim, DXM 3 free base was $\mathrm{N}$-demethylated via the $2^{\prime}, 2^{\prime}, 2^{\prime}$-trichloroethylcarbamate intermediate 14 , as originally described by Peet et al. $[18,20]$, to produce nor-DXM 15. Reductive amination, particularly using sodium triacetoxyborohydride (STABH) as a reductant, represents a mild and chemoselective method for the $\mathrm{N}$-alkylation of primary and secondary amines [36]. Tertiary amines 16am were thus synthesized from nor-DXM 15 using $n$-alkyl, cycloalkyl, heteroaryl, and bis-heteroaryl aldehydes in presence of STABH, with moderate to high yields (33-99\%) (Figure 4). To decipher the influence of the protonation state of the nitrogen atom on the antiplasmodial activity of ent-morphinans-taking into account that tazopsine $1, \mathrm{~N}$ cyclopentyl-tazopsine 2, DXM 3, DX 12, nor-DXM 15, and its $N$-alkyl derivatives 16a$\mathbf{m}$ are to be fully protonated at physiological $\mathrm{pH}$ values-two compounds $\mathbf{1 7}$ and $\mathbf{1 8}$ with a neutral or constitutively positive charge on the nitrogen atom, respectively, were synthesized. Cpd. 17, corresponding to the exact amide congener of the amine 16d for the purpose of precise SAR comparison, was prepared by reacting nor-DXM 15 with cyclopropanecarbonyl chloride in the presence of triethylamine, with a $75 \%$ yield. On the other hand, the quaternary ammonium 18 was obtained with a $82 \%$ yield by reacting DXM 3 free base with methyl iodide (Figure 4). 


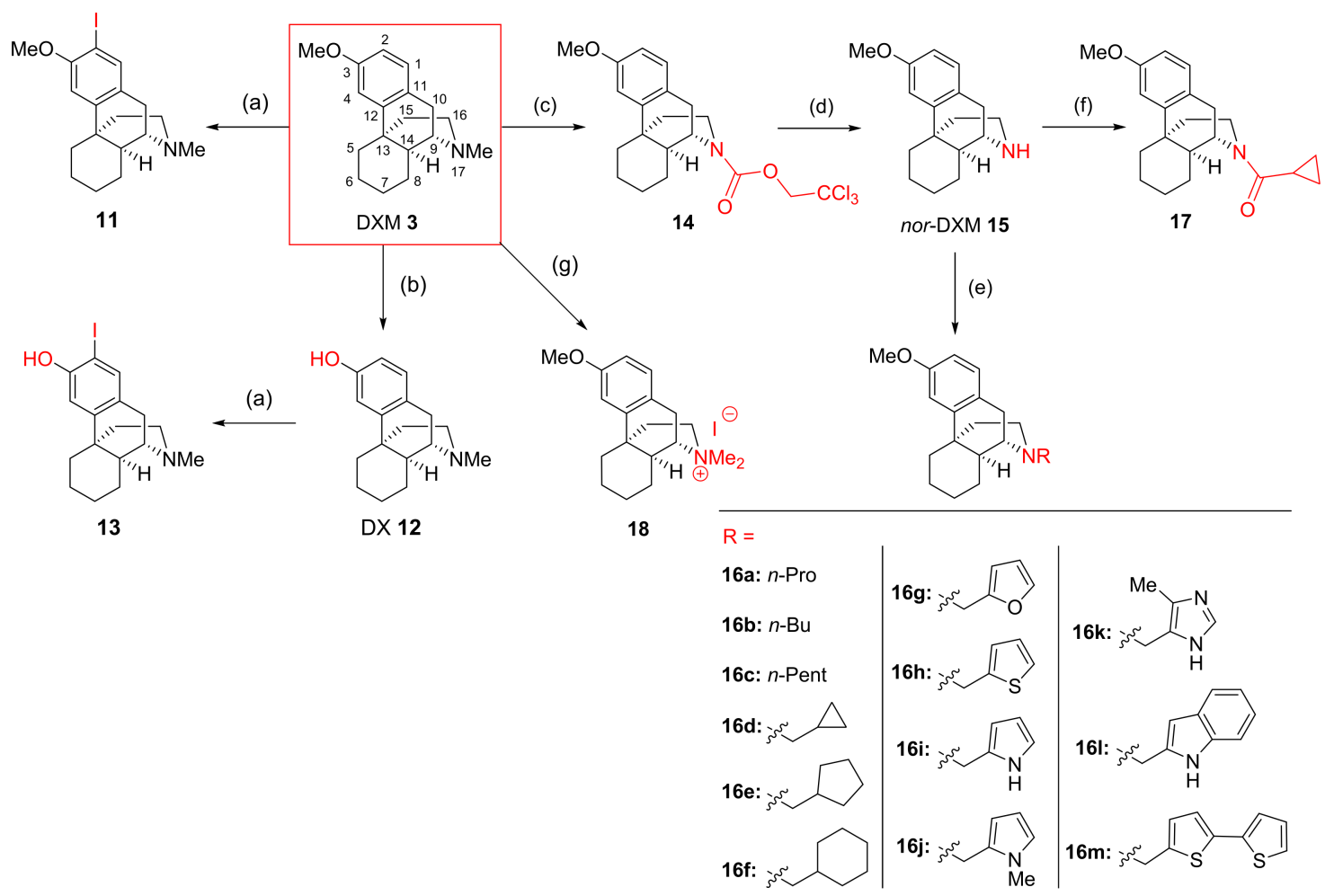

Figure 4. Semisynthetic access to DXM derivatives 11-18 for SAR generation (morphine numbering). (a) NIS (1.15 eq), $p$-TsOH (1.8 eq) (91\% 11, 87\% 13); (b) $\mathrm{HBr} 48 \%$, reflux overnight (91\%); (c) $2^{\prime}, 2^{\prime}, 2^{\prime}$-trichloroethylchloroformate (1.1 eq), toluene, reflux, $2 \mathrm{~h}(92 \%)$; (d) Zn powder (3 eq), AcOH, r. t., 1 h (47\%); (e) aldehyde (1.1 eq), DMF, r. t., 10 min then STABH (2 eq), r. t. (33-97\%); (f) cyclopropanecarbonyl chloride (1.1 eq), $\mathrm{CH}_{2} \mathrm{Cl}_{2}, \mathrm{Et}_{3} \mathrm{~N}, 0^{\circ} \mathrm{C}$ to r. t, $1.5 \mathrm{~h}(75 \%)$; (g) MeI, rt, $2 \mathrm{~h}(82 \%)$.

\subsection{In Vitro Pre-Screening against P. berghei Liver Stages}

The antiplasmodial activity against parasite liver stages of the obtained 21-compound Library was first pre-screened using $\mathrm{PHH}$ infected by $P$. berghei expressing the green fluorescent protein (PHH-Pb-GFP). This model has the advantage of being less expensive and more accessible than $P$. falciparum hepatic stages due to the routine production of $P$. berghei sporozoites in our laboratory, and also exploits the possible use of PHH to grow $P$. berghei, in order to be representative of $P$. falciparum-host cell interactions in a prescreening context. Compound activity was assessed by two criteria: (i) the number of parasites developing within PHH (exoerythrocytic forms, EEFs, Figure 5A), and (ii) the size of parasites $\left(\mu \mathrm{m}^{2}\right)$ normalized to untreated controls (Figure $5 \mathrm{~B}$ ). False positives, due to compound toxicity against host $\mathrm{PHH}$, were excluded by normalizing the parasite number to the nuclei number of untreated controls. At the highest concentration $(20 \mu \mathrm{M})$, viability of $\mathrm{PHH}$ was $>80 \%$ for the least active compounds, and 50-60\% for the most active compounds. Four activity profiles could be categorized from the 21-compound library pre-screening in terms of EEF number (Figure 5A), namely: (i) inactive compounds (2-I-DX 13, 14, 16f, 16h, 16j, 17, 18); (ii) low-activity compounds with similar inhibitory potency to DXM 3 (DX 12, Nor-DXM 15, 16b, 16c, 16d, 16e, 16k, and 16m); (iii) active compounds (2-I-DXM 11, 16a, 16g, and 161); (iv) one highly active compound (16i). These activity ranges were respectively characterized by: (i) a high EEF number at $20 \mu \mathrm{M}$ (the maximal concentration of the range), (ii) a low EEF number at $20 \mu \mathrm{M}$, (iii) a low EEF number at $10 \mu \mathrm{M}$, and finally (iv) a low EEF number at $1 \mu \mathrm{M}$. We observed the same trend in the plot of parasite size (Figure 5B), with the delineation of cpd. 16i exhibiting an important size effect at $1 \mu \mathrm{M}$, followed by cpds. 11, 16c, 16e, 16g, and 161 at $10 \mu \mathrm{M}$. In the light of these results, we focused on compounds possessing an inhibitory activity between 1-10 $\mu \mathrm{M}$, and established an amplified cut-off test at the arbitrary concentration of $7 \mu \mathrm{M}$ to validate the above-described 
categories of inhibitors (Figure 6). This evaluation permitted to identify which compounds inhibited liver stage parasite development (e.g., leading to a 50\% reduction in EEF number normalized to the drug-free controls). Five molecules were thus validated in the cut-off test, i.e., cpds. 16c, 16d, 16i, 161, and 17 (Figure 6A). In addition, 16e was intermediate and susceptible to exhibiting interesting activity. A low EEF size effect was observed for cpds. 16g, 16h, 16i, 161, and 16m, although none of these molecules inhibited parasite size by $50 \%$ or more (Figure 6B). Regarding the substitution of DXM 3 or DX 12 with iodine on the C-2 position, only 2-I-DXM 11 exhibited increased potency relative to the parent compound, whereas 2-I-DX 13 was inactive (Figure 5). Interestingly, amine 16d, and its amide congener 17, displayed similar levels of inhibitory activity against $P$. berghei liver stages (Figures 5 and 6). This suggested that the presence of an electrodonating doublet on the nitrogen atom, rather than that of a protonated doublet, was an important feature for the interaction of ent-morphinans with their biochemical target(s). However, this behavior was contradictory to that observed in the tazopsine series, whereby $N$-acetylation abolished activity (Table 2). Nevertheless, this trend in the DXM series was reinforced by the lower activity of the quaternary ammonium 18 compared to DXM 3 (Figure 5). This showed that, despite the intrinsically charged state of most bioactive DXM derivatives at physiological $\mathrm{pH}$ (with the exception of cpd. 17), the presence of a permanently charged nitrogen atom was detrimental to the antiplasmodial activity.

A

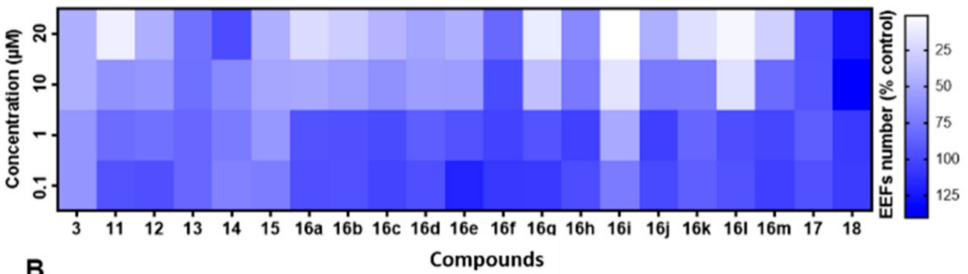

B Compounds

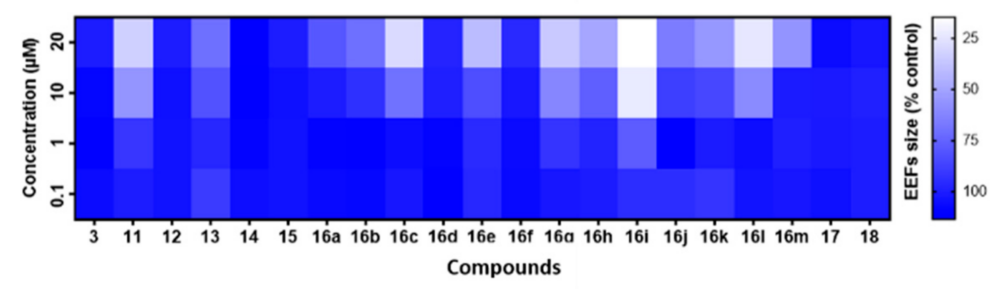

Figure 5. Normalized dose-response heatmap in PHH-Pb-GFP regarding EEF number (A) and EEF size (B) at $0.1,1,10$, or $20 \mu \mathrm{M}$ of the indicated compounds. These results were obtained from four technical replicates. 

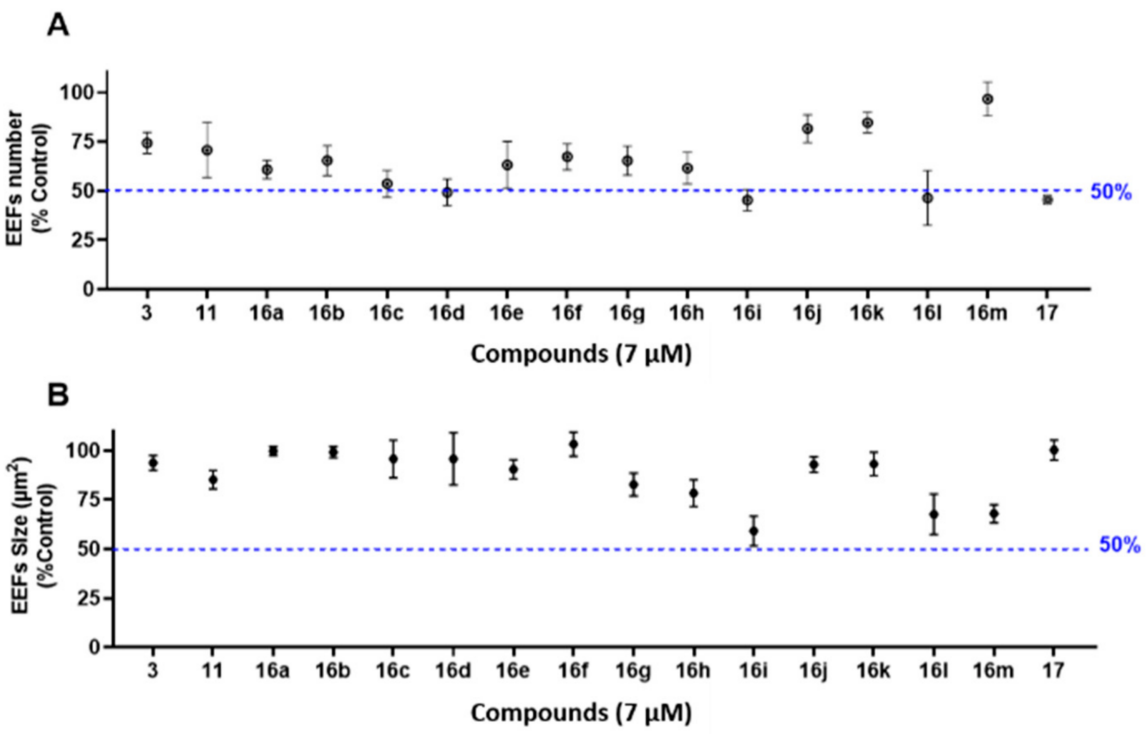

Figure 6. Normalized EEF number (A) and EEF size (B) in PHH-Pb-GFP at the arbitrary cut-off value of $7 \mu \mathrm{M}$ for the indicated compounds. Each point $(\bullet)$ represents the average EEF number of four technical replicates. The error bars represent the interval of variability between EEF numbers. These results were obtained from four technical replicates.

\subsection{In Vitro Screening against P. falciparum Liver and Asexual Blood Stages}

2-I-DXM 11, 16a $\left(\mathrm{R}=\mathrm{N}\right.$-n-propyl), 16g $\left(\mathrm{R}=\mathrm{N}\right.$-2'-furanylmethyl), 16i $\left(\mathrm{R}=\mathrm{N}-2^{\prime}\right.$ pyrrolylmethyl), and $161\left(\mathrm{R}=\mathrm{N}-2^{\prime}\right.$-indolylmethyl) displayed the best inhibitory effects in the $P$. berghei pre-screening and were thus selected for screening against both the liver and blood stages of the human parasite P. falciparum and the determination of their $\mathrm{IC}_{50}$ values. $\mathrm{PQ}$ and chloroquine $(\mathrm{CQ})$ were used as reference drugs against the liver and blood stages, respectively. Compound toxicity against host $\mathrm{PHH}$ was excluded by normalizing the parasite number to the nuclei number of untreated controls. At $10 \mu \mathrm{M}$, the viability of $\mathrm{PHH}$ was ca. $100 \%$ for the least active compounds, and $60-70 \%$ for the most active compounds. The observed increase in the antiplasmodial activity of 2-I-DXM 11 compared to DXM 3 against $P$. berghei liver stages was confirmed against $P$. falciparum, the latter being 4 -fold more active than the parent compound (Table 4). Regarding the pre-screened $\mathrm{N}$-modified analogues $16 \mathbf{a}, \mathbf{1 6} \mathbf{g}, \mathbf{1 6} \mathbf{i}$, and $\mathbf{1 6 1}$, these were active against $P$. falciparum liver stages in the low micromolar/submicromolar range and displayed significantly lower $\mathrm{IC}_{50}$ values than both parent compounds, i.e., the natural hit tazopsine 1 and DXM 3 (Tables 2 and 4). The most active compound was the $N-2^{\prime}$-pyrrolylmethyl derivative 16i, which strongly inhibited the development of parasite liver stages, being 10-fold more active than tazopsine 1 (Table 2) and 20-fold more active than DXM 3 (Table 4). Strikingly, cpd. 16i showed a superimposable inhibitory potency to the antimalarial drug PQ against $P$. falciparum liver stages ( $\mathrm{IC}_{50}$ values of $0.76 \pm 0.13 \mu \mathrm{M}$ and $0.75 \pm 0.15 \mu \mathrm{M}$, respectively). Cpd. 16i was also very active on the parasite blood stages $\left(\mathrm{IC}_{50}\right.$ values of $\left.2.1 \pm 0.4 \mu \mathrm{M}\right)$, again similarly to $\mathrm{PQ}$, which has reported $\mathrm{IC}_{50}$ values against the $P$. falciparum $3 \mathrm{D} 7$ strain in the 1-20 $\mu \mathrm{M} \mathrm{IC}_{50}$ range [24,37]. With the exception of $\mathbf{1 6 i}$, only $\mathbf{1 6 1}$ showed activity in the low micromolar range against $P$. falciparum blood stages $\left(\mathrm{IC}_{50}\right.$ of $6.5 \pm 0.4 \mu \mathrm{M}$ ). Other $\mathrm{N}$-alkylated derivatives exhibited low activity against blood stages, similar to DXM 3, with $\mathrm{IC}_{50}$ values of $43-62 \mu \mathrm{M}$. However, all compounds were found to be selective for the hepatic phase of parasite development (2.8-36-fold selectivity) including DXM 3 (4.9-fold selectivity) (Table 4). 
Table 4. In vitro $\mathrm{IC}_{50}$ values of tazopsine 1, DXM 3, and selected $N$-modified derivatives against P. falciparum liver (NF54) and blood (3D7) stages, as well as liver phase selectivity values (S). IC 50 values are the mean of four technical replicates for $\mathrm{PHH}$ and three technical replicates for HE. $\mathrm{R}$ identities for $N$-substituted DXM 3 derivatives refer to Figure 4. HE: human erythrocytes; NT: not tested; NA: not applicable.

\begin{tabular}{|c|c|c|c|c|}
\hline \multirow{2}{*}{ Cpds. } & \multirow{2}{*}{$\mathbf{R}$} & \multicolumn{2}{|c|}{$\mathrm{IC}_{50}(\mu \mathrm{M})$} & \multirow{2}{*}{$S$} \\
\hline & & PfNF54-PHH & Pf3D7-HE & \\
\hline Tazopsine 1 & NA & $7.88 \pm 3.05$ & $4.07 \pm 0.87$ & 0.5 \\
\hline DXM 3 & $\mathrm{Me}$ & $15.59 \pm 1.19$ & $76.5 \pm 0.9$ & 4.9 \\
\hline 11 & NA & $4.10 \pm 2.77$ & $61.7 \pm 5.3$ & 15.0 \\
\hline $16 a$ & $n$-propyl & $2.25 \pm 3.03$ & $43.3 \pm 2.3$ & 19.2 \\
\hline $16 \mathrm{~g}$ & 2'-furanylmethyl & $1.56 \pm 0.59$ & $56.2 \pm 2.7$ & 36.0 \\
\hline $16 \mathrm{i}$ & $2^{\prime}$-pyrrolylmethyl & $0.76 \pm 0.13$ & $2.1 \pm 0.4$ & 2.8 \\
\hline 161 & 2'-indolylmethyl & $1.98 \pm 0.34$ & $6.5 \pm 0.4$ & 3.3 \\
\hline PQ & NA & $0.75 \pm 0.15$ & NT & NA \\
\hline CQ & NA & NT & $0.033 \pm 0.016$ & NA \\
\hline
\end{tabular}

3.6. In Vitro Screening against P. falciparum Sexual Blood Stages

To explore the activity profile of the new hit cpd. 16i against other developmental stages of P. falciparum, we tested it against early (stages I-II) and late (stage V) gametocytes, the last being responsible for the transmission of $P$. falciparum malaria. Cpd. 16i was found to be fairly effective against both gametocyte stages, with activities in the high micromolar range ( $\mathrm{IC}_{50}$ values of $18.5 \mu \mathrm{M}$ and $13.2 \mu \mathrm{M}$, respectively), whereas DXM 3 was completely inactive $\left(\mathrm{IC}_{50}>100 \mu \mathrm{M}\right)$ (Figure 7).

A

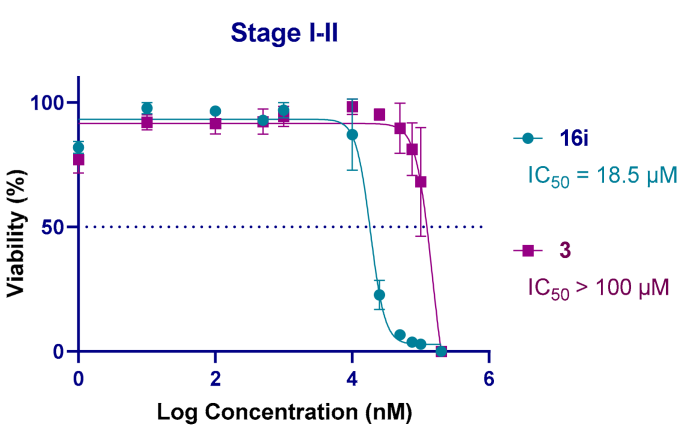

B

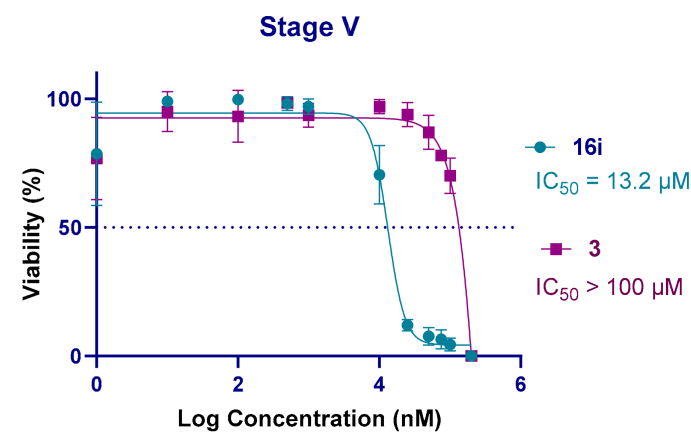

Figure 7. Inhibitory plots and $\mathrm{IC}_{50}$ values of DXM 3 (purple) and cpd. 16i (cyan) against $P$. falciparum early (stages I-II) (A) and late (stage V) (B) gametocytes in vitro. $\mathrm{IC}_{50}$ values are the mean of three technical replicates and three biological replicates. Error bars represent standard deviation. 


\section{Conclusions}

The rapid spread of artemisinin-resistant P. falciparum strains across Southeast Asia [5] and independent increase of $\mathrm{k} 13$ polymorphisms in Africa [38,39], which culminated in clinical artemisinin resistance recently being detected in the continent [6-8], is a dramatic continuum of the drug resistance history of malarial parasites. This situation underscores the need for alternative chemotherapeutic strategies beyond pursuing novel drugs to eliminate blood parasites, prone to acquiring resistance mutations. In this context, the antiplasmodial alkaloid tazopsine 1, introduced in the late 2000s, is active against both pre-erythrocytic and erythrocytic P. falciparum stages, and constitutes the precursor of the in vivo prophylactic compound $\mathrm{N}$-cyclopentyl-tazopsine 2 . However, the development of the tazopsines through biosourcing or synthetic strategies was difficult. Aiming to overcome these limitations, we managed to simplify the natural alkaloid tazopsine 1 -the same principle also applying to the related sinococuline 5-and to extract a "naked" entmorphinan antiplasmodial pharmacophore in the form of the antitussive drug DXM 3. In particular, substitutions at the level of rings $B$ and $C$ proved non-essential, whereas those affecting ring A (particularly at C-2) were of potential relevance. Despite its limited potential for a repurposing against malaria, DXM 3 exhibited a significant level of bioactivity in vitro, that was only 2 -fold lower than that of tazopsine $\mathbf{1}$ against $P$. falciparum liver stages. Capitalizing on strengthened ring D SARs, a rapid diversification of DXM 3 into $\mathrm{N}$-modified derivatives readily led to improved analogues. Amongst those, the hit cpd. $\mathrm{N}$ 2 '-pyrrolylmethyl-nor-DXM 16i exhibited a 10-fold and 20-fold increase in activity against $P$. falciparum liver stages relative to tazopsine 1 and DXM 3, respectively, and showed similar activity than the reference drug primaquine against parasite liver and blood stages. In the context of prophylactic antimalarial drug discovery, cpd. 16i was more selective for the parasite liver phase than tazopsine $\mathbf{1}(\mathrm{S}=2.8 \mathrm{vs} .0 .5)$ in addition to having a stronger bioactivity than the natural alkaloid. Moreover, cpd. 16i had a significant effect on the viability of stage I-II and V P. falciparum gametocytes compared to the inactive DXM 3. These results warrant further mechanistic investigation into the new hit cpd. 16i regarding causal prophylaxis (i.e., early sterilization of sporozoites) and possible pan-activity against multiple parasite stages. In addition, the benefit of C-2 substitution suggests it may be possible to further optimize derivatives in the hit series by means of ring A modifications.

Author Contributions: The manuscript was written with contributions from all authors. All authors have read and agreed to the published version of the manuscript.

Funding: We thank the Doctoral School of Paris University (ED 563, MTCI) for financial support (PhD fellowship to A.K.). L.V. was supported by the OrganoMal project funded by the French National Agency for Research (ANR) (ANR-CE17-0035-0011) and a CIFRE ANRT allocation N²019/0153. LB was supported by the European Union's Horizon 2020 Research and Innovation Program under grant agreement $\mathrm{N}^{\circ} 733273$.

Institutional Review Board Statement: Not applicable.

Informed Consent Statement: Not applicable.

Data Availability Statement: Not applicable.

Acknowledgments: We thank Jocelyne Roman for helping with the evaluation of the prophylactic activity of DXM 3 in mice infected with P. berghei, Thierry Houpert for his invaluable help for the breeding of Anopheles mosquitoes, and Jérôme Clain for relevant suggestions regarding the manuscript. This work benefited from equipment and services from the CELIS cell culture core facility (Institut du Cerveau et de la Moëlle Epinière, Paris), a platform supported through ANR grants number ANR-10-IAIHU-06 and ANR-11-INBS-0011-NeurATRIS. IDMIT infrastructure is supported by the French government Program d'Investissements d'Avenir (PIA) under grant number ANR-11INBS-0008 (INBS IDMIT). This work was partly supported by the OrganoMal Project funded by the French National Agency for Research (ANR) (ANR-CE17-0035-0011) and the PARAFRAP LabEx of the French Parasitology Alliance For Health Care (ANR-11-LABX-0024). LVA was supported by a 
CIFRE ANRT allocation $\mathrm{N}^{\circ} 181029 \mathrm{~A} 20$. We are particularly grateful to David Akbar for his valuable assistance regarding automated fluorescence imaging.

Conflicts of Interest: The authors declare no conflict of interest.

\begin{abstract}
Abbreviations
$\mathrm{Ac}_{2} \mathrm{O}$ : acetic anhydride; $\mathrm{AcOH}$, acetic acid; $\mathrm{ACT}$, artemisinin combination therapies; ARTDs, artemisinin derivatives; $\mathrm{Bu}$, butane; cpd., compound; $\mathrm{CNS}$, central nervous system; $\mathrm{CQ}$, chloroquine; DAPI, 4',6-diamidino-2-phenylindole; DMF, N,N-dimethylformamide; DX, dextrorphan; DXM, dextromethorphan; EEFs, exoerythrocytic forms; $\mathrm{Et}_{3} \mathrm{~N}$, triethyl amine; $\mathrm{Et}_{2} \mathrm{O}$, diethyl ether; EtOAc, ethyl acetate; GFP, green florescent protein; HRMS, high-resolution mass spectra; $\mathrm{IC}_{50}$, half-maximal inhibitory concentration; IE, infected erythrocytes; Me, methyl; $\mathrm{MeCN}$, acetonitrile; MeI, methyl iodide; $\mathrm{MeOH}$, methanol, NIS, $N$-iodosuccinimide; NMDA, $N$-methyl-D-aspartate; NMR, nuclear magnetic resonance; Pb, Plasmodium berghei; Pent, pentane; Pf, Plasmodium falciparum; Py, P. yoelii; $\mathrm{PHH}$, primary human hepatocyte, $\mathrm{PMH}$, primary mice hepatocytes; $\mathrm{PQ}$, primaquine; Pro, propane; QND, quinidine; rpm, rotation per minute; r. t., room temperature; SGs, salivary glands; STABH, sodium triacetoxyborohydride; TLC, thin-layer chromatography.
\end{abstract}

\title{
References
}

1. WHO. World Malaria Report; WHO: Geneva, Switzerland, 2019.

2. Ritchie, H.; Roser, M. Causes of Death. Our World in Data 2018. Available online: https:/ / ourworldindata.org/causes-of-death (accessed on 28 December 2021).

3. Bhatt, S.; Weiss, D.J.; Cameron, E.; Bisanzio, D.; Mappin, B.; Dalrymple, U.; Battle, K.E.; Moyes, C.L.; Henry, A.; Eckhoff, P.A.; et al. The effect of malaria control on Plasmodium falciparum in Africa between 2000 and 2015. Nature 2015, 526, 207-211. [CrossRef] [PubMed]

4. $\quad$ Phillips, M.A.; Burrows, J.N.; Manyando, C.; van Huijsduijnen, R.H.; Van Voorhis, W.C.; Wells, T.N.C. Malaria. Nat. Rev. Dis. Primers 2017, 3, 17050. [CrossRef] [PubMed]

5. Imwong, M.; Suwannasin, K.; Kunasol, C.; Sutawong, K.; Mayxay, M.; Rekol, H.; Smithuis, F.M.; Hlaing, T.M.; Tun, K.M.; van der Pluijm, R.W.; et al. The spread of artemisinin-resistant Plasmodium falciparum in the Greater Mekong subregion: A molecular epidemiology observational study. Lancet Infect. Dis. 2017, 17, 491-497. [CrossRef]

6. Ikeda, M.; Kaneko, M.; Tachibana, S.I.; Balikagala, B.; Sakurai-Yatsushiro, M.; Yatsushiro, S.; Takahashi, N.; Yamauchi, M.; Sekihara, M.; Hashimoto, M.; et al. Artemisinin-Resistant Plasmodium falciparum with High Survival Rates, Uganda, $2014-2016$. Emerg. Infect. Dis. 2018, 24, 718-726. [CrossRef] [PubMed]

7. Uwimana, A.; Umulisa, N.; Venkatesan, M.; Svigel, S.S.; Zhou, Z.; Munyaneza, T.; Habimana, R.M.; Rucogoza, A.; Moriarty, L.F.; Sandford, R.; et al. Association of Plasmodium falciparum kelch13 R561H genotypes with delayed parasite clearance in Rwanda: An open-label, single-arm, multicentre, therapeutic efficacy study. Lancet Infect. Dis. 2021, 21, 1120-1128. [CrossRef]

8. Balikagala, B.; Fukuda, N.; Ikeda, M.; Katuro, O.T.; Tachibana, S.I.; Yamauchi, M.; Opio, W.; Emoto, S.; Anywar, D.A.; Kimura, E.; et al. Evidence of Artemisinin-Resistant Malaria in Africa. N. Engl. J. Med. 2021, 385, 1163-1171. [CrossRef]

9. Witkowski, B.; Amaratunga, C.; Khim, N.; Sreng, S.; Chim, P.; Kim, S.; Lim, P.; Mao, S.; Sopha, C.; Sam, B.; et al. Novel phenotypic assays for the detection of artemisinin-resistant Plasmodium falciparum malaria in Cambodia: In-vitro and ex-vivo drug-response studies. Lancet Infect. Dis. 2013, 13, 1043-1049. [CrossRef]

10. Bloland, P.B.; Ettling, M. Making malaria-treatment policy in the face of drug resistance. Ann. Trop. Med. Parasitol. 1999, 93, 5-23. [CrossRef]

11. Derbyshire, E.R.; Prudencio, M.; Mota, M.M.; Clardy, J. Liver-stage malaria parasites vulnerable to diverse chemical scaffolds. Proc. Natl. Acad. Sci. USA 2012, 109, 8511-8516. [CrossRef]

12. Kato, N.; Comer, E.; Sakata-Kato, T.; Sharma, A.; Sharma, M.; Maetani, M.; Bastien, J.; Brancucci, N.M.; Bittker, J.A.; Corey, V.; et al. Diversity-oriented synthesis yields novel multistage antimalarial inhibitors. Nature 2016, 538, 344-349. [CrossRef]

13. Le Manach, C.; Dam, J.; Woodland, J.G.; Kaur, G.; Khonde, L.P.; Brunschwig, C.; Njoroge, M.; Wicht, K.J.; Horatscheck, A.; Paquet, T.; et al. Identification and Profiling of a Novel Diazaspiro[3.4]octane Chemical Series Active against Multiple Stages of the Human Malaria Parasite Plasmodium falciparum and Optimization Efforts. J. Med. Chem. 2021, 64, 2291-2309. [CrossRef] [PubMed]

14. Carraz, M.; Jossang, A.; Franetich, J.F.; Siau, A.; Ciceron, L.; Hannoun, L.; Sauerwein, R.; Frappier, F.; Rasoanaivo, P.; Snounou, G.; et al. A plant-derived morphinan as a novel lead compound active against malaria liver stages. PLoS Med. 2006, 3, e513. [CrossRef] [PubMed]

15. Carraz, M.; Jossang, A.; Rasoanaivo, P.; Mazier, D.; Frappier, F. Isolation and antimalarial activity of new morphinan alkaloids on Plasmodium yoelii liver stage. Bioorg. Med. Chem. 2008, 16, 6186-6192. [CrossRef] 
16. Zahari, A.; Cheah, F.K.; Mohamad, J.; Sulaiman, S.N.; Litaudon, M.; Leong, K.H.; Awang, K. Antiplasmodial and antioxidant isoquinoline alkaloids from Dehaasia longipedicellata. Planta Med. 2014, 80, 599-603. [CrossRef] [PubMed]

17. Jakobsson, J.E.; Riss, P.J. Transition metal free, late-stage, regiospecific, aromatic fluorination on a preparative scale using a KF/crypt-222 complex. RSC Adv. 2018, 8, 21288-21291. [CrossRef]

18. Jozwiak, K.; Targowska-Duda, K.M.; Kaczor, A.A.; Kozak, J.; Ligeza, A.; Szacon, E.; Wrobel, T.M.; Budzynska, B.; Biala, G.; Fornal, E.; et al. Synthesis, in vitro and in vivo studies, and molecular modeling of N-alkylated dextromethorphan derivatives as non-competitive inhibitors of alpha3beta4 nicotinic acetylcholine receptor. Bioorg. Med. Chem. 2014, 22, 6846-6856. [CrossRef] [PubMed]

19. Bouyer, G.; Barbieri, D.; Dupuy, F.; Marteau, A.; Sissoko, A.; N’Dri, M.E.; Neveu, G.; Bedault, L.; Khodabux, N.; Roman, D.; et al. Plasmodium falciparum sexual parasites regulate infected erythrocyte permeability. Commun. Biol. 2020, 3, 726. [CrossRef] [PubMed]

20. Peet, N.P. N-Demethylation of dextromethorphan. J. Pharm. Sci. 1980, 69, 1447-1448. [CrossRef] [PubMed]

21. Cevenini, L.; Camarda, G.; Michelini, E.; Siciliano, G.; Calabretta, M.M.; Bona, R.; Kumar, T.R.; Cara, A.; Branchini, B.R.; Fidock, D.A.; et al. Multicolor bioluminescence boosts malaria research: Quantitative dual-color assay and single-cell imaging in Plasmodium falciparum parasites. Anal. Chem. 2014, 86, 8814-8821. [CrossRef]

22. Bergmann, C.; van Loon, W.; Habarugira, F.; Tacoli, C.; Jager, J.C.; Savelsberg, D.; Nshimiyimana, F.; Rwamugema, E.; Mbarushimana, D.; Ndoli, J.; et al. Increase in Kelch 13 Polymorphisms in Plasmodium falciparum, Southern Rwanda. Emerg. Infect. Dis. 2021, 27, 294-296. [CrossRef] [PubMed]

23. Uwimana, A.; Legrand, E.; Stokes, B.H.; Ndikumana, J.M.; Warsame, M.; Umulisa, N.; Ngamije, D.; Munyaneza, T.; Mazarati, J.B.; Munguti, K.; et al. Emergence and clonal expansion of in vitro artemisinin-resistant Plasmodium falciparum kelch13 R561H mutant parasites in Rwanda. Nat. Med. 2020, 26, 1602-1608. [CrossRef]

24. Cabrera, M.; Cui, L. In Vitro Activities of Primaquine-Schizonticide Combinations on Asexual Blood Stages and Gametocytes of Plasmodium falciparum. Antimicrob. Agents Chemother. 2015, 59, 7650-7656. [CrossRef]

25. Borch, R.F.; Bernstein, M.D.; Dupont Durst, H. Cyanohydridoborate anion as a selective reducing agent. J. Am. Chem. Soc. 1971, 93, 2897-2904. [CrossRef]

26. Renia, L.; Mattei, D.; Goma, J.; Pied, S.; Dubois, P.; Miltgen, F.; Nussler, A.; Matile, H.; Menegaux, F.; Gentilini, M.; et al. A Malaria Heat-Shock-Like Determinant Expressed on the Infected Hepatocyte Surface Is the Target of Antibody-Dependent Cell-Mediated Cytotoxic Mechanisms by Nonparenchymal Liver-Cells. Eur. J. Immunol. 1990, 20, 1445-1449. [CrossRef]

27. Bosson-Vanga, H.; Franetich, J.F.; Soulard, V.; Sossau, D.; Tefit, M.; Kane, B.; Vaillant, J.C.; Borrmann, S.; Muller, O.; DereuddreBosquet, N.; et al. Differential activity of methylene blue against erythrocytic and hepatic stages of Plasmodium. Malar. J. 2018, 17, 143. [CrossRef]

28. Manzoni, G.; Briquet, S.; Risco-Castillo, V.; Gaultier, C.; Topcu, S.; Ivanescu, M.L.; Franetich, J.F.; Hoareau-Coudert, B.; Mazier, D.; Silvie, $\mathrm{O}$. A rapid and robust selection procedure for generating drug-selectable marker-free recombinant malaria parasites. Sci. Rep. 2014, 4, 4760. [CrossRef] [PubMed]

29. Desjardins, R.E.; Canfield, C.J.; Haynes, J.D.; Chulay, J.D. Quantitative assessment of antimalarial activity in vitro by a semiautomated microdilution technique. Antimicrob. Agents Chemother. 1979, 16, 710-718. [CrossRef]

30. Oh, S.R.; Agrawal, S.; Sabir, S.; Taylor, A. Dextromethorphan; StatPearls: Treasure Island, FL, USA, 2021.

31. Shin, E.J.; Bach, J.H.; Lee, S.Y.; Kim, J.M.; Lee, J.; Hong, J.S.; Nabeshima, T.; Kim, H.C. Neuropsychotoxic and neuroprotective potentials of dextromethorphan and its analogs. J. Pharmacol. Sci. 2011, 116, 137-148. [CrossRef]

32. Annoura, T.; Chevalley, S.; Janse, C.J.; Franke-Fayard, B.; Khan, S.M. Quantitative analysis of Plasmodium berghei liver stages by bioluminescence imaging. Methods Mol. Biol. 2013, 923, 429-443. [CrossRef]

33. Schadel, M.; Wu, D.; Otton, S.V.; Kalow, W.; Sellers, E.M. Pharmacokinetics of dextromethorphan and metabolites in humans: Influence of the CYP2D6 phenotype and quinidine inhibition. J. Clin. Psychopharmacol. 1995, 15, 263-269. [CrossRef] [PubMed]

34. Capon, D.A.; Bochner, F.; Kerry, N.; Mikus, G.; Danz, C.; Somogyi, A.A. The influence of CYP2D6 polymorphism and quinidine on the disposition and antitussive effect of dextromethorphan in humans. Clin. Pharmacol. Ther. 1996, 60, 295-307. [CrossRef]

35. Gorski, J.C.; Jones, D.R.; Wrighton, S.A.; Hall, S.D. Characterization of dextromethorphan N-demethylation by human liver microsomes. Contribution of the cytochrome P450 3A (CYP3A) subfamily. Biochem. Pharmacol. 1994, 48, 173-182. [CrossRef]

36. Abdel-Magid, A.F.; Carson, K.G.; Harris, B.D.; Maryanoff, C.A.; Shah, R.D. Reductive Amination of Aldehydes and Ketones with Sodium Triacetoxyborohydride. Studies on Direct and Indirect Reductive Amination Procedures(1). J. Org. Chem. 1996, 61, 3849-3862. [CrossRef] [PubMed]

37. Adjalley, S.H.; Johnston, G.L.; Li, T.; Eastman, R.T.; Ekland, E.H.; Eappen, A.G.; Richman, A.; Sim, B.K.; Lee, M.C.; Hoffman, S.L.; et al. Quantitative assessment of Plasmodium falciparum sexual development reveals potent transmission-blocking activity by methylene blue. Proc. Natl. Acad. Sci. USA 2011, 108, E1214-E1223. [CrossRef] [PubMed]

38. Le Nagard, H.; Vincent, C.; Mentre, F.; Le Bras, J. Online analysis of in vitro resistance to antimalarial drugs through nonlinear regression. Comput. Methods Prog. Biomed. 2011, 104, 10-18. [CrossRef] [PubMed]

39. Kaddouri, H.; Nakache, S.; Houze, S.; Mentre, F.; Le Bras, J. Assessment of the drug susceptibility of Plasmodium falciparum clinical isolates from africa by using a Plasmodium lactate dehydrogenase immunodetection assay and an inhibitory maximum effect model for precise measurement of the 50-percent inhibitory concentration. Antimicrob. Agents Chemother. 2006, 50, 3343-3349. 